\title{
A Posteriori Error Control for Three Typical Force-Based Atomistic-to-Continuum Coupling Methods for an Atomistic Chain
}

\author{
Hao Wang ${ }^{1, *}$, Shaohui $\mathrm{Liu}^{2}$ and Feng Yang ${ }^{3}$ \\ ${ }^{1}$ School of Mathematics, Sichuan University, No. 24 South Section One, \\ Chengdu, 610065, China \\ ${ }^{2}$ Department of Applied Mathematics and Statistics, Stony Brook University, \\ Stony Brook, New York, 11794-3600, U.S \\ ${ }^{3}$ College of Mathematical Science, Beijing University, Zhongguancun Road 1, \\ Beijing, 100000, China
}

Received 28 July 2017; Accepted (in revised version) 28 April 2018

\begin{abstract}
We consider the problem of a posteriori error estimates and adaptivity for three typical force-based atomistic-to-continuum coupling methods. Combining the residual and the stability estimates, we derive computable a posteriori error estimators for the three methods in the energy norm and formulate adaptive algorithms using these estimators. Our numerical experiments show optimal convergence rates of these algorithms. The efficiency of the estimators are also demonstrated numerically.
\end{abstract}

AMS subject classifications: 65N12, 65N15, 70C20, 82D25

Key words: Atomistic-to-continuum coupling, a posteriori error estimate, adaptivity, force based method.

\section{Introduction}

Atomistic-to-continuum (a/c) coupling methods, also known as quasicontinuum (QC) methods, are a class of of multiscale methods for coupling an atomistic model of a solid with a continuum model which is often obtained by the Cauchy-Born approximation. The atomistic model is used in certain regions of interest, such as crystal defects and their neighbourhoods, to gain a good accuracy while a continuum model is applied to reduce the cost of the computation in the elastic far fields. We refer to $[25,26,32]$ for perfect reviews for such methods.

There are two major types of a/c coupling methods, namely the energy-based methods and the force-based methods. The energy-based methods approximate the energy of the atomistic system by a coupling energy and essentially solve energy minimization problems

${ }^{*}$ Corresponding author. Email addresses: wangh@scu.edu.cn (H. Wang), shaohui.liu@stonybrook.edu (S. H. Liu), yang_feng@pku.edu.cn (F. Yang) 
$[12,17-20,30,35,38,40]$. However, the original energy-based (QCE) method, despite its simple formulation, exhibits spurious force at the coupling interface which reduces the accuracy of computing the deformation and the lattice stability $[7,8,28,33]$ while the improved energy-based methods have the difficulty of the reconstruction of the energy of the interface atoms $[35,36,38,39]$. On the other hand, the force-based methods directly solve equilibrium equations of the coupling forces at each degree of freedom $[6,14,23]$ and possess better accuracy with simple constructions. However the original force-based (QCF) method has certain stability issues which make the determination of the lattice stability challenging. The stability is then improved by blending the atomistic force with the continuum force in certain regions so that the blended equation becomes positive definite [16] which gives the blended QCF (BQCF) method. Such improvement is also achieved by changing the formulation of the problem to a stress-based variational formulation which gives the stress-based a/c (SAC) method [23].

Considerable efforts have been devoted to the a priori analysis of the force-based methods $[4,6,10,14,16,22,24,27]$, especially the (in-)stability of these methods as mentioned earlier. Meanwhile, comparatively little research has been carried out to the $a$ posteriori error estimates and adaptivity of the force-based methods. The existing literature, to the best knowledge of the authors, only consider energy-based methods [1-3, 34, 37, 41, 42].

The current work aims to provide a first step for the a posteriori error estimates and adaptivity of the force-based methods. We derive the residual based error estimators for three prototype force-based methods, namely the QCF, the SAC and the BQCF methods, following the framework provided in [34]. We consider a 1D periodic next-nearest-neighbour multi-body interaction atomistic system and its force-based approximations. Though in a 1D setting, the system we consider and the numerical experiments we do preserve many important aspects in higher dimensions which we believe are valuable contributions to this field.

The outline of the paper is listed below.

In Section 2, we introduce the atomistic model problem, the three force-based a/c coupling methods and the notation that will be used throughout the paper. We note that some of the setting and notation follows from [23].

In Section 3, we derive the residual estimates for the three methods in a discrete negative Sobolev norm. We see that the residual estimate of the QCF method can be given as a special case of the BQCF method.

In Section 4, we give the a posteriori stability analysis through a different approach compared with that in [34]. In Section 5, we combine the residual estimates and the stability to give a posteriori error estimators for the deformation gradient.

In Section 6, we develop adaptive algorithms according to the error estimators and present numerical examples to illustrate the performances. In particular, we consider what we call the model adaptivity problem using the residual approach.

We note that we will use a/c (short name for atomistic-to-continuum) and QC (short name for quasicontinuum) liberally since they have the same meaning at least in the present work. 


\section{The Atomistic model and its force-based QC approximations}

\subsection{Atomistic model}

We formulate our problem on an infinite lattice $\mathscr{L}_{\#}=\mathbb{Z}$ and look for the solution of our atomistic problem in the space of $2 N$-periodic displacements

$$
\mathscr{U}^{\varepsilon}:=\left\{u: \mathscr{L}_{\#} \rightarrow \mathbb{R}^{\mathbb{Z}}: u_{\ell+2 N}=u_{\ell} \text { and } u_{0}=0\right\} .
$$

Since the solution is periodic, we only consider a specific period of the lattice denoted by

$$
\mathscr{L}:=\{-N+1,-N+2, \cdots, N-1, N\} .
$$

Let $\varepsilon=1 / N$ then $\Omega=[-1,1]$ is essentially the domain we consider. The corresponding admissible set of deformations is defined by

$$
\mathscr{Y}^{\varepsilon}:=\left\{y: \mathscr{L}_{\#} \rightarrow \mathbb{R}^{\mathbb{Z}}: y_{\ell}=\varepsilon F \ell+v_{\ell}, v \in \mathscr{U}^{\varepsilon}\right\},
$$

where $\varepsilon=1 / N$. We note that $\mathscr{U}^{\varepsilon}$ and $\mathscr{Y}^{\varepsilon}$ are identified with the $\mathscr{P}_{1}$ functions with respect to the canonic mesh associated with the lattice $\mathscr{T}_{\varepsilon}=\left\{T_{\ell}^{\varepsilon}\right\}_{\ell \in \mathbb{Z}}$ where $T_{\ell}^{\varepsilon}=[(\ell-1) \varepsilon, \ell \varepsilon]$.

For $v \in \mathbb{R}^{\mathbb{Z}}$, we define $v^{\prime}=\left\{v_{\ell}^{\prime}\right\}_{(\ell \in \mathbb{Z})}, v^{\prime \prime}=\left\{v_{\ell}^{\prime \prime}\right\}_{(\ell \in \mathbb{Z})}$ by

$$
v_{\ell}^{\prime}=\frac{v_{\ell}-v_{\ell-1}}{\varepsilon} \quad \text { and } \quad v_{\ell}^{\prime \prime}=\frac{v_{\ell+1}^{\prime}-v_{\ell}^{\prime}}{\varepsilon} .
$$

We assume that the atomistic system is modeled by a next-nearest-neighbour multi-body potential and the internal energy in one period under $y \in \mathscr{Y}^{\varepsilon}$ is given by

$$
\mathscr{E}^{\mathrm{a}}(y):=\varepsilon \sum_{\ell \in \mathscr{L}} V\left(D y_{\ell}\right)
$$

where $V \in C^{3}\left((0,+\infty]^{4} ; \mathbb{R}\right)$ is a general multi-body potential and

$$
D y_{\ell}:=\left(D_{1} y_{\ell}, D_{2} y_{\ell}, D_{-1} y_{\ell}, D_{-2} y_{\ell}\right)
$$

for which

$$
D_{1} y_{\ell}:=\frac{y_{\ell+1}-y_{\ell}}{\varepsilon}, \quad D_{2} y_{\ell}:=\frac{y_{\ell+2}-y_{\ell}}{\varepsilon}, \quad D_{-1} y_{\ell}:=\frac{y_{\ell-1}-y_{\ell}}{\varepsilon}, \quad D_{-2} y_{\ell}:=\frac{y_{\ell-2}-y_{\ell}}{\varepsilon} .
$$

Assuming that the external forces are dead loads which is representable by $g \in \mathscr{U}^{\varepsilon}$, the total energy (per period) under a deformation $y \in \mathscr{Y}$ is given by

$$
\mathscr{E}_{\text {tot }}^{\mathrm{a}}(y)=\mathscr{E}^{\mathrm{a}}(y)-\langle g, y\rangle_{\varepsilon},
$$

where $\langle g, y\rangle_{\epsilon}=\sum_{\ell \in \mathscr{L}} g_{\ell} y_{\ell}$, and the solution we seek for the atomistic problem is:

$$
\text { Find } y_{\mathrm{a}} \in \mathscr{Y}^{\varepsilon}, \quad \text { s.t. } y_{\mathrm{a}} \in \operatorname{argmin} \mathscr{E}_{\text {tot }}^{\mathrm{a}}(\mathscr{Y}) \text {. }
$$


If $y_{\mathrm{a}}$ is a solution to (2.4), it then satisfies the first-order criticality condition

$$
\left(f^{\mathrm{a}}\left(y_{\mathrm{a}}\right), v\right)_{\varepsilon}=(g, v)_{\varepsilon} \quad \forall v \in \mathscr{U}^{\varepsilon},
$$

where $f^{\mathrm{a}}(y)=\left\{f^{\mathrm{a}}(y)_{\ell}\right\}_{\ell \in \mathbb{Z}}$ for $y \in \mathscr{Y}^{\varepsilon}$ are defined by

$$
f_{\ell}^{\mathrm{a}}(y):=\frac{1}{\varepsilon} \frac{\partial \mathscr{E}^{\mathrm{a}}(y)}{\partial y_{\ell}}=\frac{1}{\varepsilon}\left[\sigma_{\ell+1}^{\mathrm{a}}(y)-\sigma_{\ell}^{\mathrm{a}}(y)\right] \quad \forall \ell \in \mathbb{Z},
$$

for which

$$
\begin{aligned}
\sigma_{\ell}^{\mathrm{a}}(y):=\partial_{1} V & \left(D y_{\ell-1}\right)-\partial_{-1} V\left(D y_{\ell}\right)+\partial_{2} V\left(D y_{\ell-2}\right) \\
& +\partial_{2} V\left(D y_{\ell-1}\right)-\partial_{-2} V\left(D y_{\ell}\right)-\partial_{-2} V\left(D y_{\ell+1}\right)
\end{aligned}
$$

is the atomistic stress tensor. Using the periodic boundary condition, it is easy to show that (c.f. [23]) the left hand side of the variational problem (2.5) is actually the first variation of the atomistic energy, i.e.,

$$
\left(f^{\mathrm{a}}(y), v\right)_{\varepsilon}=\left\langle\delta \mathscr{E}^{\mathrm{a}}(y), v\right\rangle=\varepsilon \sum_{\ell \in \mathscr{L}} \sigma_{\ell}^{\mathrm{a}} v_{\ell}^{\prime} .
$$

\subsection{The local $\mathrm{QC}$ approximation}

We define the Cauchy-Born energy functional for a given $y \in W^{1, \infty}(\Omega ; \mathbb{R})$ by

$$
\mathscr{E}^{\mathrm{c}}(y):=\int_{\Omega} W(\nabla y) \mathrm{d} x,
$$

where $W(F):=V(F, 2 F,-F,-2 F) \in C^{3}(0,+\infty)$ is the Cauchy-Born stored energy density [35]. The local QC approximation is the P1-finite element discretization of $\mathscr{E}$.

To formulate the local QC approximation, we partition $\Omega$ by choosing a small number of, say $K$, lattice sites as the finite element nodes and construct the mesh $\mathscr{T}_{h}=\left\{T_{k}\right\}_{k=1}^{K}$ on $\Omega$ with the following restrictions and definitions:

1. The nodes are identified with the indices of the lattice sites by $\ell:\{1, \cdots, K\} \rightarrow \mathscr{L}$ such that $\ell_{k}:=\ell(k)$ is the index of the lattice site which is also the $k^{\prime}$ th node in $\mathscr{T}_{h}$.

2. $\mathscr{T}_{h}=\left\{T_{k}\right\}_{k=1}^{K}$ where $T_{k}:=\left[x_{k-1}, x_{k}\right]$ and $x_{k}=\varepsilon \ell_{k}$ for all $k=1, \cdots K$. The length of $T_{k}$ is given by $h_{T_{k}}:=\left|T_{k}\right|=x_{k}-x_{k-1}$.

We then define the coarse-grained space of displacement as

$$
\mathscr{U}^{h}:=\left\{u_{h} \in \mathscr{P}_{1}\left(\mathscr{T}_{h}\right): u_{h}(x+1)=u_{h}(x) \text { and } u_{h}(0)=0\right\},
$$

and the coarse-grained admissible set of deformation as

$$
\mathscr{Y}^{h}:=\left\{y_{h} \in \mathscr{P}_{1}\left(\mathscr{T}_{h}\right): y_{h}=F x+u_{h}(x), u_{h} \in \mathscr{U}_{h}\right\}
$$


where $\mathscr{P}_{1}\left(\mathscr{T}_{h}\right)$ denotes the space of continuous piecewise affine functions with respect to $\mathscr{T}_{h}$. We note that by the construction of $\mathscr{T}^{h}, \mathscr{U}^{h} \subset \mathscr{U}^{\varepsilon}$ and hence any $u_{h}$ is canonically identified with its nodal interpolant $I_{\varepsilon} u_{h} \in \mathscr{T}_{\varepsilon}$ with respect to $\mathscr{T}_{\varepsilon}$, and so is any $y_{h}$, for example, $u_{\ell_{k}}^{h}:=u_{h}\left(x_{k}\right)$.

The internal energy of the local QC approximation under $y_{h} \in \mathscr{Y}^{h}$ is given by

$$
\mathscr{E}^{c}(y)=\int_{\Omega} W\left(\nabla y_{h}\right) \mathrm{d} x=\sum_{k=1}^{K} h_{T_{k}} W\left(\left.\nabla y_{h}\right|_{T_{k}}\right) .
$$

If we approximate the external energy by the trapezium rule and define $\widetilde{\omega}_{k}=T_{k} \cup T_{k+1}$ and $H_{k}=\frac{1}{2}\left|\widetilde{\omega}_{k}\right|$, we obtain the total local QC energy

$$
\mathscr{E}_{\text {tot }}^{\mathrm{c}}(y)=\sum_{k=1}^{K} h_{T_{k}} W\left(\left.\nabla y_{h}\right|_{T_{k}}\right)-\left\langle g, y_{h}\right\rangle_{h}
$$

where $\left\langle g, y_{h}\right\rangle_{h}:=\sum_{k=1}^{K} H_{k} g_{\ell_{k}} y_{h}\left(x_{k}\right)$, and the solution we seek for the local QC problem is

$$
\text { Find } y_{\mathrm{qcl}} \in \mathscr{Y}^{h}, \quad \text { s.t. } y_{\mathrm{qcl}} \in \operatorname{argmin} \mathscr{E}_{\mathrm{tot}}^{\mathrm{c}}\left(\mathscr{Y}^{h}\right) \text {. }
$$

Similar to the atomistic problem, if $y_{\mathrm{qcl}}$ is a solution to (2.14), it then satisfies

$$
\left(F^{\mathrm{c}}\left(y_{\mathrm{qcl}}\right), v_{h}\right)_{h}=\left(g, v_{h}\right)_{h} \quad \forall v_{h} \in \mathscr{U}^{h},
$$

where $F^{\mathrm{c}}\left(y_{h}\right)=\left\{F_{k}^{\mathrm{c}}\left(y_{h}\right)\right\}_{k \in \mathbb{Z}}$ for $y_{h} \in \mathscr{Y}^{h}$ are given by

$$
F_{k}^{\mathrm{c}}\left(y_{h}\right):=\frac{1}{H_{k}} \frac{\partial \mathscr{E}^{\mathrm{c}}\left(y_{h}\right)}{\partial y_{h}\left(x_{k}\right)}=\frac{1}{H_{k}}\left[\sigma_{T_{k+1}}^{\mathrm{c}}\left(y_{h}\right)-\sigma_{T_{k}}^{\mathrm{c}}\left(y_{h}\right)\right] \quad \forall \ell \in \mathbb{Z}
$$

for which

$$
\sigma_{T_{k}}^{\mathrm{c}}\left(y_{h}\right):=W^{\prime}\left(\nabla y_{h} \mid T_{k}\right)
$$

is the continuum stress tensor. Again it is easy to show that (c.f. [23]) the left hand side of the variational problem (2.15) is the first variation of the continuum energy, i.e.,

$$
\left(F^{\mathrm{c}}\left(y_{h}\right), v\right)_{h}=\left\langle\delta \mathscr{E}^{\mathrm{c}}\left(y_{h}\right), v_{h}\right\rangle=\left.\sum_{k=1}^{K} h_{T_{k}} \sigma_{T_{k}}^{\mathrm{c}} \nabla v\right|_{T_{k}} .
$$

\subsection{The force-based QC methods}

In this section, we introduce three force-based QC methods which are the original forcebased QC method (QCF), the blended force-based QC method (BQCF) and the stress-based QC method (SAC).Though the force-based QC method can be considered as a special case of the blended force-based QC method, we nevertheless single it out as it provides the fundamental idea and motivation for the other two methods. 


\subsubsection{The QCF method}

We begin by decomposing the lattice $\mathscr{L}$ into $\mathscr{A}$ and $\mathscr{C}$, where $\mathscr{A}$ denotes the set of lattice sites inside which full atomistic accuracy is required, and $\mathscr{C}:=\mathscr{L} \backslash \mathscr{A}$ denotes the remaining lattice sites. We then partition the computational domain $\Omega$ into $\Omega_{\mathrm{a}}$ and $\Omega_{\mathrm{c}}$ according to the decomposition of $\mathscr{L}$ so that each lattice site in $\mathscr{A}$ is a node in $\mathscr{T}_{h}$. For simplicity of presentation, we assume only one atomistic region $\Omega_{\mathrm{a}}$ exists. Then there exists $1<\underline{K}<\bar{K}<K$ such that

$$
\mathscr{A}=\left\{\ell_{\underline{K}}, \ell_{\underline{K}+1}, \cdots, \ell_{\bar{K}}\right\},
$$

and $\Omega_{\mathrm{a}}=\left[\varepsilon \ell_{\underline{K}}, \varepsilon \ell_{\bar{K}}\right]$. We define the sets of the indices of the nodes in the period, the nodes in the atomistic region and the nodes in the continuum region respectively by

$$
\mathscr{K}:=\{1,2, \cdots, K\}, \quad \mathscr{K}^{\mathrm{a}}:=\{\underline{K}, \underline{K}+1, \cdots, \bar{K}\} \quad \text { and } \quad \mathscr{K}^{\mathrm{c}}:=\mathscr{K} \backslash \mathscr{K}^{\mathrm{a}} .
$$

To simplify the analysis and presentation, we require that the two atoms in the continuum region which are adjacent to the atomistic region are also nodes in $\mathscr{T}_{h}$, i.e., $\ell_{\underline{K}}-1=\ell_{\underline{K}-1}$ and $\ell_{\bar{K}}+1=\ell_{\bar{K}+1}$. This essentially means that we put an extra layer of atoms around the atomistic region, see [14] for a similar setting. We denote the periodic extensions of $\mathscr{A}$ and $\mathscr{C}$ by $\mathscr{A}_{\#}$ and $\mathscr{C}_{\#}$.

The QCF force $F^{\mathrm{qcf}}\left(y_{h}\right)=\left\{F_{k}^{\mathrm{qcf}}\left(y_{h}\right)\right\}_{k \in \mathbb{Z}}$ for $y_{h} \in \mathscr{Y}^{h}$ are given by

$$
F_{k}^{\mathrm{c}}\left(y_{h}\right)= \begin{cases}f_{\ell_{k}}^{\mathrm{a}}\left(y_{h}\right), & k \in \mathscr{K}^{\mathrm{a}}, \\ F_{k}^{\mathrm{c}}\left(y_{h}\right), & k \in \mathscr{K}^{\mathrm{c}},\end{cases}
$$

and the QCF method is defined by the following nonlinear variational problem:

$$
\text { Find } y_{\mathrm{qcf}} \in \mathscr{Y}^{h}, \quad \text { s.t. } \quad\left(F^{\mathrm{qcf}}\left(y_{\mathrm{qcf}}\right), v_{h}\right)_{h}=\left(g, v_{h}\right)_{h}, \quad \forall v_{h} \in \mathscr{Y}^{h} \text {. }
$$

By summation by parts, the left hand side of the QCF variational problem (2.20) for $y_{h} \in$ $\mathscr{Y}^{h}$ can be written as (c.f. [24, Section 4])

$$
\begin{aligned}
\left(F^{\mathrm{qcf}}\left(y_{\mathrm{qcf}}\right), v_{h}\right)_{h}= & \sum_{k \in \mathscr{K}^{a}} \varepsilon \sigma_{\ell_{k}}^{\mathrm{a}}\left(v_{h}^{\prime}\right)_{\ell_{k}}+\left.\sum_{k \in \mathscr{K}^{\mathrm{c}}} h_{T_{k}} \sigma_{k}^{\mathrm{c}} \nabla v_{h}\right|_{T_{k}} \\
& -v_{\underline{\ell}_{\underline{K}-1}}^{h}\left(\sigma_{T_{\underline{K}}^{\mathrm{c}}}^{\mathrm{c}}-\sigma_{\ell_{\underline{K}}}^{\mathrm{a}}\right)+v_{\ell_{\bar{K}}}^{h}\left(\sigma_{T_{\bar{K}+1}^{\mathrm{c}}}^{\mathrm{c}}-\sigma_{\ell_{\bar{K}+1}}^{\mathrm{a}}\right) .
\end{aligned}
$$

Remark 2.1. It was proved in [6] that the QCF force is nonconservative, i.e., there does not exist a potential whose gradient with respect to the deformation equals to the QCF force, which results in the fact that (2.21) is not a variation of an energy functional as opposed to the atomistic variational problem (2.8) and the continuum variational problem (2.18).

Compared with any energy-based a/c coupling method, the QCF method possesses a higher order accuracy $[5,6,14,21,27]$. However, it was shown in $[4,9,10]$ that the QCF operator lack of uniform coercivity with respect to the size of the atomistic system. Variants of the QCF method which we review in Section 2.3.2 and Section 2.3.3 appeared to tackle this issue. 


\subsubsection{The SAC method}

The first variant of the QCF method that we analyze is the stress-based a/c coupling method (the SAC method) which was developed in [23] and analyzed in detail in [24]. The idea is that we drop the interface terms on the second line of (2.21) and define the SAC stress tensor $F^{\mathrm{sac}}\left(y_{h}\right)=\left\{F^{\mathrm{sac}}\left(y_{h}\right)_{T_{k}}\right\}_{k \in \mathbb{Z}}$ such that

$$
\sigma_{T_{k}}^{\mathrm{sac}}\left(y_{h}\right)= \begin{cases}\sigma_{\ell_{k}}^{\mathrm{a}}\left(y_{h}\right), & \ell_{k} \in \mathscr{A} \\ \sigma_{T_{k}}^{\mathrm{c}}\left(y_{h}\right), & \ell_{k} \in \mathscr{C}\end{cases}
$$

and the problem we solve is to find $y_{h} \in \mathscr{Y}_{h}$ such that

$$
\begin{aligned}
\left(F^{\mathrm{sac}}\left(y_{h}\right), v_{h}\right)_{h}: & =\sum_{k \in \mathscr{K}^{\mathrm{a}}} \varepsilon \sigma_{\ell_{k}}^{\mathrm{a}}\left(y_{h}\right)\left(v_{h}^{\prime}\right)_{\ell_{k}}+\left.\sum_{k \in \mathscr{K}^{\mathrm{c}}} h_{T_{k}} \sigma_{T_{k}}^{\mathrm{c}}\left(y_{h}\right) \nabla v_{h}\right|_{T_{k}} \\
& =\left(g, v_{h}\right)_{h}, \quad \forall v_{h} \in \mathscr{U}^{h} .
\end{aligned}
$$

The main advantage of the SAC method over the QCF method is that the linearized SAC operator is positive definite in general (except possibly close to bifurcation point), which results in a direct a priori error estimates by duality in the discrete $H^{1}$-norm $[23,24]$.

\subsubsection{The BQCF method}

The second variant of the QCF method we will analyze is the blended force-based quasicontinuum method (the BQCF method) [14-16,21,27]. To formulate the BQCF method, we first introduce a blending region $\Omega_{\mathrm{b}}$ between the atomistic region and the continuum region and decompose the lattice into $\mathscr{L}=\mathscr{A} \cup \mathscr{B} \cup \mathscr{C}$ accordingly. To be precise, if we inherit the lattice decomposition in (2.19) and assume there is only one atomistic region, we can choose $\underline{L}$ and $\bar{L}$ such that $1<\underline{L}<\underline{K}<\bar{K}<\bar{L}<K$ and define

$$
\mathscr{B}:=\left\{\ell_{\underline{L}}, \ell_{\underline{L}}+1, \cdots, \ell_{\underline{K}}, \ell_{\bar{K}}, \cdots, \ell_{\bar{L}}-1, \ell_{\bar{L}}\right\},
$$

and $\Omega_{\mathrm{b}}=[\varepsilon \underline{L}, \varepsilon \underline{K}] \cup[\varepsilon \bar{K}, \varepsilon \bar{L}]$. Similar to the QCF method, we put an extra layer of atoms around the blending region, i.e., $\ell_{\underline{L}}-1=\ell_{\underline{L}-1}$ and $\ell_{\bar{L}}+1=\ell_{\bar{L}+1}$. The periodic extension of $\mathscr{B}$ is denoted by $\mathscr{B}_{\#}$.

We define a (smooth) blending function $\beta: \mathbb{Z} \rightarrow[0,1]$ such that

$$
\beta(\ell)= \begin{cases}0, & \forall \ell \in \mathscr{A}_{\#}, \\ 1, & \forall \ell \in \mathscr{C}_{\#},\end{cases}
$$

and define the BQCF force $F^{\mathrm{bqcf}}\left(y_{h}\right)=\left\{F_{k}^{\mathrm{bqcf}}\left(y_{h}\right)\right\}_{k \in \mathbb{Z}}$ for $y_{h} \in \mathscr{Y}^{h}$ by

$$
F_{k}^{\mathrm{bqcf}}(y):=\left(1-\beta\left(\ell_{k}\right)\right) f_{\ell_{k}}^{a}\left(y_{h}\right)+\beta\left(\ell_{k}\right) F_{k}^{\mathrm{c}}\left(y_{h}\right)
$$


The purpose of such construction is to 'spread' the interface effect caused by the second line of $(2.21)$ so that certain stability property holds for the BQCF method [14-16,21,27]. We usually define the blending function $\beta$ by scaling such that for $\ell \in \mathscr{B}$

$$
\beta(\ell)= \begin{cases}\hat{\beta}\left(\frac{\ell-\ell_{\underline{K}}}{\ell_{\underline{K}}-\ell_{\underline{L}}}\right), & \ell_{\underline{L}} \leq \ell \leq \ell_{\underline{K}}, \\ \hat{\beta}\left(\frac{\ell_{\bar{K}}-\ell}{\ell_{\bar{L}}-\ell_{\bar{K}}}\right), & \ell_{\bar{K}} \leq \ell \leq \ell_{\bar{L}},\end{cases}
$$

where $\hat{\beta} \in C^{3}$ is a smooth function whose definition will be given later. The BQCF is then defined by the nonlinear variational problem:

$$
\text { Find } y_{h}^{\text {bqcf }} \in \mathscr{Y}^{h} \text {, s.t. } \quad\left(F^{\mathrm{bqcf}}\left(y^{\mathrm{bqcf}}\right), v_{h}\right)_{h}=\left(g, v_{h}\right)_{h} \quad \forall v_{h} \in \mathscr{U}_{h} .
$$

We could also write the BQCF method in a divergence form using the following proposition (c.f. [32, Section 6.4]).

Proposition 2.1. Let $y_{h} \in \mathscr{Y}^{h}$; then, $\forall v_{h} \in \mathscr{U}_{h}$

$$
\left(F^{\mathrm{bqcf}}\left(y_{h}\right), v_{h}\right)_{h}=\sum_{\ell \in \mathscr{A} \cup \mathscr{B}} \varepsilon \sigma_{\ell}^{\mathrm{a}}\left(y_{h}\right)\left(v_{\mathrm{a}}^{h}\right)_{\ell}^{\prime}+\left.\sum_{k \in \mathscr{K}_{\mathrm{b}} \cup \mathscr{K}_{\mathrm{a}}} h_{T_{k}} \sigma_{T_{k}}^{\mathrm{c}}\left(y_{h}\right) \nabla v_{\mathrm{c}}^{h}\right|_{T_{k}},
$$

where $v_{\mathrm{a}}^{h}=(1-\beta) v_{h}$ and $v_{\mathrm{c}}^{h}=\beta v_{h}$ and $\mathscr{K}_{b}=\{\underline{L}, \cdots, \underline{K}\} \cup\{\bar{K}, \cdots, \bar{L}\}$.

Proof. By the property that $\beta$ ceases to zero in the atomistic region but equals to one in the continuum region and the mesh structure of the BQCF method, we have

$$
\begin{aligned}
\left(F^{\mathrm{bqcf}}\left(y_{h}\right), v_{h}\right)_{h} & =\left((1-\beta) f^{\mathrm{a}}\left(y_{h}\right)+\beta F^{\mathrm{c}}\left(y_{h}\right), v_{h}\right)_{h} \\
& =\left(f^{\mathrm{a}}(y),(1-\beta) v_{h}\right)_{\varepsilon}+\left(F^{\mathrm{c}}(y), \beta v_{h}\right)_{h} .
\end{aligned}
$$

By the smoothness of $\beta$ and the variational formulations (2.8) and (2.18), we have

$$
\begin{aligned}
& \left(F^{\mathrm{bqcf}}\left(y_{h}\right), v_{h}\right)_{h}=\sum_{\ell \in \mathscr{A} \cup \mathscr{B}} \varepsilon\left[(1-\beta(\ell)) v_{h}\right]_{\ell}^{\prime} \sigma_{\ell}^{\mathrm{a}}+\left.\sum_{k \in \mathscr{K}^{\mathrm{b}} \cup \mathscr{K}^{\mathrm{c}}} h_{T_{k}} \nabla\left(\beta\left(\ell_{k}\right) v\right)\right|_{T_{k}} \sigma_{T_{k}}^{\mathrm{c}} \\
& =\sum_{\ell \in \mathscr{A} \cup \mathscr{B}} \varepsilon\left(v_{\mathrm{a}}^{h}\right)_{\ell}^{\prime} \sigma_{\ell}^{\mathrm{a}}\left(y_{h}\right)+\left.\sum_{k \in \mathscr{K}^{\mathrm{b}} \cup \mathscr{K}^{\mathrm{c}}} h_{T_{k}} \nabla v_{\mathrm{c}}^{h}\right|_{T_{k}} \sigma_{T_{k}}^{\mathrm{c}}\left(y_{h}\right),
\end{aligned}
$$

which is the stated result.

Remark 2.2. We note that (2.25) reduces to (2.21) when $\mathscr{B}=\left\{\ell_{\underline{K}}-1, \ell_{\underline{K}}\right\} \cup\left\{\ell_{\bar{K}}, \ell_{\bar{K}}+1\right\}$ and we may thus consider the QCF method as a special case of the BQCF method. 


\subsection{Further formulation and notation}

We introduce some formulation and notation that would accommodate our analysis in subsequent sections.

We define the set of indices of lattice sites inside and on the right boundary of the element $T_{k}$ by

$$
\mathscr{L}_{T_{k}}:=\left\{\ell_{k-1}+1, \cdots, \ell_{k}\right\} .
$$

By the 1D setting of our problem and the definition of $\mathscr{Y}^{\varepsilon}$ and $\mathscr{Y}^{h}$, we have

$$
\left.\nabla v_{h}\right|_{T}=\frac{\varepsilon}{h_{T}} \sum_{\ell \in \mathscr{L}_{T}} v_{\ell}^{\prime}, \quad \forall v \in \mathscr{Y}^{h} .
$$

Consequently the following equation holds that

$$
\left.h_{T_{k}} \sigma_{T_{k}}^{\mathrm{c}}\left(y_{h}\right) \nabla v_{h}\right|_{T_{k}}=\varepsilon \sum_{\ell \in \mathscr{L}_{T_{k}}} \sigma_{\ell}^{\mathrm{c}}\left(y_{h}\right)\left(v^{h}\right)_{\ell}^{\prime}, \quad \forall k \in \mathscr{K}^{\mathrm{b}} \cup \mathscr{K}^{\mathrm{c}},
$$

where the continuum stress tensor associated with the lattice $\sigma_{\ell}^{\mathrm{c}}\left(y_{h}\right)$ is identical to $\sigma_{T_{k}}^{\mathrm{c}}\left(y_{h}\right)$ for $\ell \in \mathscr{L}_{T_{k}}$. This identity is very useful in our analysis.

We define the set which contains the indices of the nodes in the continuum region that are not adjacent to the interface nodes as

$$
\mathscr{K}^{\mathrm{c}}:= \begin{cases}\mathscr{K}^{\mathrm{c}} \backslash\{\underline{K}-1, \bar{K}+1\} & \text { for the QCF method, } \\ \mathscr{K}^{\mathrm{c}} \backslash\{\underline{L}-1, \bar{L}+1\} & \text { for the BQCF method. }\end{cases}
$$

We also define the set which contains the indices of the elements in the 'central continuum region' as

$$
\mathscr{K}_{\mathscr{T}_{h}}^{\mathrm{c}}:= \begin{cases}\mathscr{K}^{\mathrm{c}} \backslash\{\underline{K}-1, \bar{K}+2\} & \text { for the QCF method, } \\ \mathscr{K}^{\mathrm{c}} \backslash\{\underline{L}-1, \bar{L}+2\} & \text { for the BQCF method. }\end{cases}
$$

Let $\mathscr{D}$ be a subset of $\mathbb{Z}$. For a vector $v \in \mathbb{R}^{\mathbb{Z}}$, we define

$$
\|v\|_{\ell_{\varepsilon}^{p}(\mathscr{D})}:= \begin{cases}\left(\sum_{\ell \in \mathscr{D}} \varepsilon\left|v_{\ell}\right|^{p}\right)^{1 / p}, & 1 \leq p<\infty \\ \max _{\ell \in \mathscr{D}}\left|v_{\ell}\right|, & p=\infty\end{cases}
$$

If the label $\mathscr{D}$ is omitted, it means $\mathscr{D}=\{-N+1, \cdots, N\}$.

We define the first order discrete derivatives $v_{\ell}^{\prime}:=\left(v_{\ell}-v_{\ell-1}\right) / \varepsilon$ for $v \in \mathscr{U}^{\varepsilon}$ and equip the space $\mathscr{U}^{\varepsilon}$ with the discrete Sobolev norm

$$
\|v\|_{\mathscr{U}^{1,2}}:=\left\|v^{\prime}\right\|_{\ell_{\varepsilon}^{2}} \quad \text { for } v \in \mathscr{U}^{\varepsilon} .
$$

The norm on the dual space $\left(\mathscr{U}^{\varepsilon}\right)^{*}$ is defined by

$$
\|T\|_{\mathscr{U}^{-1,2}}:=\sup _{\substack{v \in \mathscr{U}^{\varepsilon} \\\|v\|_{\mathscr{U}^{1,2}}=1}} T[v] .
$$




\section{Residual estimates}

\subsection{Framework of the residual estimates}

We first introduce the framework which essentially follows those from [34,41,42]. We define the residual operator $R: \mathscr{Y}^{h} \rightarrow\left(\mathscr{U}^{\varepsilon}\right)^{*}$ by

$$
\begin{aligned}
R[v] & =\left\langle\delta \mathscr{E}^{\mathrm{a}}\left(y_{\mathrm{ac}}\right), v\right\rangle-\langle g, v\rangle_{\varepsilon} \\
& =\left(\left\langle\delta \mathscr{E}^{\mathrm{a}}\left(y_{\mathrm{ac}}\right), v\right\rangle-\langle g, v\rangle_{\varepsilon}\right)-\left(\left(S^{\mathrm{ac}}\left(y_{\mathrm{ac}}\right), v\right)_{h}-(g, v)_{h}\right) \\
& =\left(\left\langle\delta \mathscr{E}^{\mathrm{a}}\left(y_{\mathrm{ac}}\right), v\right\rangle-\left(S^{\mathrm{ac}}\left(y_{\mathrm{ac}}\right), v\right)_{h}\right)+\left((g, v)_{h}-\langle g, v\rangle_{\varepsilon}\right) \\
& =: R_{\mathrm{int}}[v]+R_{\mathrm{ext}}[v],
\end{aligned}
$$

where $S^{\mathrm{ac}} \in\left\{\sigma^{\mathrm{sac}}, F^{\mathrm{qcf}}, F^{\mathrm{bqcf}}\right\}$ and $y_{\mathrm{ac}}$ are the corresponding a/c solutions, and we call $R_{\text {int }}[v]$ the internal residual and $R_{\text {ext }}[v]$ the external residual. Here we consider $\left(S^{\text {ac }}, \cdot\right)_{h}$ as operators on $\mathscr{U}^{\varepsilon}$ whose detailed definitions will be given for the corresponding methods. We will bound the two different types of residuals separately where the internal residual has different formulations for the SAC and the (B)QCF methods.

\subsection{Estimate of the internal residual for SAC}

We begin with the residual estimate for the SAC method whose analysis is the most straightforward one among the three. For the sake of the simplicity of notation, we assume that the size of the element whose index is in $\mathscr{K}_{\mathscr{T}_{h}}^{\mathrm{c}}$ is larger than or equal to $6 \varepsilon$ in the following theorem.

Theorem 3.1 (Elementwise internal residual estimate for SAC). Let $y_{\mathrm{ac}}$ be a solution of the $S A C$ model (2.22), $\min \left(y_{\mathrm{ac}}\right)_{\ell}^{\prime}>0$ and $R_{\mathrm{int}}^{\mathrm{sac}}:=\left\langle\delta \mathscr{E}^{\mathrm{a}}\left(y_{\mathrm{ac}}\right), v\right\rangle-\left(\sigma^{\mathrm{sac}}\left(y_{\mathrm{ac}}\right), v\right)_{h}$; then with the assumption of the size of the elements made just before the theorem, the internal residual is then estimated by

$$
\left\|R_{\text {int }}^{\mathrm{sac}}\right\|_{\mathscr{U}^{-1,2}} \leq\left\{\sum_{k \in \mathscr{K}_{\mathscr{T}_{h}}^{\mathrm{c}}}\left(\eta_{T_{k}}^{\mathrm{sac}}\right)^{2}\right\}^{\frac{1}{2}}:=\eta_{\mathrm{int}}^{\mathrm{sac}},
$$

where the elementwise upper bound of the internal residual is given by

$$
\begin{aligned}
& \eta_{T_{k}}^{\mathrm{sac}}= \begin{cases}\left\{\frac{1}{2} \varepsilon\left[\sum_{\ell=\ell_{k-1}-2}^{\ell_{k-1}+3}\left(R_{\ell}^{\mathrm{int}}\right)^{2}+\sum_{\ell=\ell_{k}-2}^{\ell_{k}+3}\left(R_{\ell}^{\mathrm{int}}\right)^{2}\right]\right\}^{\frac{1}{2}}, & k \in \mathscr{\mathscr { K }}_{\mathscr{T}_{h}}^{\mathrm{c}}, \\
\left\{\frac{1}{2} \varepsilon\left[\sum_{\ell=\ell_{k-1}-2}^{\ell_{k-1}+3}\left(R_{\ell}^{\mathrm{int}}\right)^{2}\right]+\varepsilon\left[\sum_{\ell=\ell_{k}-2}^{\ell_{k}}\left(R_{\ell}^{\mathrm{int}}\right)^{2}\right]\right\}^{\frac{1}{2}}, & k=\underline{K}-1, \\
\left\{\varepsilon\left[\sum_{\ell=\ell_{k-1}}^{\ell_{k-1}+3}\left(R_{\ell}^{\mathrm{int}}\right)^{2}\right]+\frac{1}{2} \varepsilon\left[\sum_{\ell=\ell_{k}-2}^{\ell_{k}+3}\left(R_{\ell}^{\mathrm{int}}\right)^{2}\right]\right\}^{\frac{1}{2}}, & k=\bar{K}+2,\end{cases} \\
& R_{\ell}^{\mathrm{int}}:=\sigma_{\ell}^{\mathrm{a}}\left(y_{\mathrm{ac}}\right)-\sigma_{\ell}^{\mathrm{c}}\left(y_{\mathrm{ac}}\right),
\end{aligned}
$$

are the internal residual associated with the lattice. 
Proof. By the variational formulation of the atomistic model (2.8) and the SAC model (2.22), and the identity (2.28), we have

$$
\begin{aligned}
R_{\mathrm{int}}^{\mathrm{sac}}[v] & =\varepsilon \sum_{\ell \in \mathscr{C}} \sigma_{\ell}^{\mathrm{a}}\left(y_{\mathrm{ac}}\right) v_{\ell}^{\prime}-\left.\sum_{k \in \mathscr{K}^{\mathrm{c}}} h_{T_{k}} \sigma_{T_{k}}^{\mathrm{c}}\left(y_{\mathrm{ac}}\right) \nabla v_{h}\right|_{T_{k}} \\
& =\sum_{k \in \mathscr{K}^{\mathrm{c}} \mathrm{c}} \varepsilon \sum_{\ell=\ell_{k}-2}^{\ell_{k}+3} R_{\ell}^{\mathrm{int}} v_{\ell}^{\prime}+\varepsilon \sum_{\ell=\ell_{\underline{\underline{K}}-1}-2}^{\ell_{\underline{K}-1}} R_{\ell}^{\mathrm{int}} v_{\ell}^{\prime}+\varepsilon \sum_{\ell=\ell_{\bar{K}+1}}^{\ell_{\bar{K}+1}+3} R_{\ell}^{\mathrm{int}} v_{\ell}^{\prime} \\
& \leq\left\{\sum_{k \in \mathscr{K}^{\mathrm{c}} \mathrm{c}} \varepsilon \sum_{\ell=\ell_{k}-2}^{\ell_{k}+3}\left(R_{\ell}^{\mathrm{int}}\right)^{2}+\varepsilon \sum_{\ell=\ell_{\underline{\underline{K}}-1}-2}^{\ell^{\underline{K}-1}}\left(R_{\ell}^{\mathrm{int}}\right)^{2}+\varepsilon \sum_{\ell=\ell_{\bar{K}+1}}^{\ell_{\bar{K}+1}+3}\left(R_{\ell}^{\mathrm{int}}\right)^{2}\right\}^{\frac{1}{2}}\left\|v^{\prime}\right\|_{\ell_{\varepsilon}^{2}},
\end{aligned}
$$

where we have used the fact that $\sigma_{\ell}^{\mathrm{a}}\left(y_{\mathrm{ac}}\right)=\sigma_{\ell}^{\mathrm{c}}\left(y_{\mathrm{ac}}\right)$ for $\ell \in \mathscr{L}_{T_{k}} \backslash\left[\left\{\ell_{k-1}+1, \ell_{k-1}+2, \ell_{k-1}+\right.\right.$ $\left.3\} \cup\left\{\ell_{k}-2, \ell_{k}-1, \ell_{k}\right\}\right]$. Regrouping the residuals with respect to nodes and elements, we obtain the stated results.

In the case without coarse-graining, i.e., in the case that we choose $\mathscr{U}^{h} \equiv \mathscr{U}^{\varepsilon}$ and $\mathscr{Y}^{h} \equiv \mathscr{Y}^{\varepsilon}$, a nodewise internal residual estimate is given in the following corollary whose proof is straight forward and thus omitted.

Corollary 3.1 (Nodewise internal residual estimate for SAC). Let $y_{\mathrm{ac}}$ be a solution of the SAC model (2.22) with the solution spaces $\mathscr{U}^{h} \equiv \mathscr{U}^{\varepsilon}$ and $\mathscr{Y}^{h} \equiv \mathscr{Y}^{\varepsilon}$. With the same assumption of $y_{\mathrm{ac}}$ and the same definition of $R_{\mathrm{int}}^{\mathrm{sac}}$ in Theorem 3.1, the internal residual is estimated by

$$
\left\|R_{\mathrm{int}}^{\mathrm{sac}}\right\|_{\mathscr{U}^{-1,2}} \leq\left\{\sum_{\ell \in \mathscr{C}}\left(\tilde{\eta}_{\ell}^{\mathrm{sac}}\right)^{2}\right\}^{\frac{1}{2}}:=\eta_{\mathrm{int}}^{\mathrm{sac}}
$$

where the nodewise upper bound of the internal residual is simply given by

$$
\left(\tilde{\eta}_{\ell}^{\mathrm{sac}}\right)^{2}:=\frac{1}{5} \sum_{\ell-2}^{\ell+2}\left(\eta_{\ell}^{\mathrm{sac}}\right)^{2},
$$

and $\eta_{\ell}^{\mathrm{sac}}:=\varepsilon R_{\ell}^{\mathrm{int}}$

Remark 3.1. We will use the result in Corollary 3.1 and its counterpart in Section 3.3 in our numerical experiments to do the model adaptivity problem. We take the average of the residual contributions around $\ell$ because each $R_{\ell}^{\text {int }}$ is affected by the atoms with indices $\ell-2$ to $\ell+2$.

\subsection{Estimate of the internal residual for BQCF}

We then derive the internal residual of the BQCF methods where the blending function presents in the error estimator. Again, in the following theorem, we assume the sizes of the elements in the continuum region to be larger than or equal to $6 \varepsilon$ as we did in theorem 3.1 . 
Theorem 3.2 (Elementwise internal residual estimate for BQCF). Let $y_{\mathrm{ac}}$ be a solution of the BQCF model (2.24), $\min \left(y_{\mathrm{ac}}\right)_{\ell}^{\prime}>0$ and $R_{\mathrm{int}}^{\mathrm{bqcf}}:=\left\langle\delta \mathscr{E}^{\mathrm{a}}\left(y_{\mathrm{ac}}\right), v\right\rangle-\left(F^{\mathrm{bqcf}}\left(y_{\mathrm{ac}}\right), v\right)_{h}$; then with the same assumption of the size of the elements as that in Theorem 3.1, the internal residual is estimated by

$$
\left\|R_{\text {int }}^{\mathrm{bqcf}}\right\|_{\mathscr{U}^{-1,2}} \leq\left\{\sum_{k \in \mathscr{K}_{\mathscr{T}_{h}}^{\mathrm{c}}}\left(\eta_{T_{k}}^{\mathrm{bqcf}}\right)^{2}\right\}^{\frac{1}{2}}:=\eta_{\text {int }}^{\mathrm{bqcf}},
$$

where the elementwise upper bound of the internal residual is given by

$$
\eta_{T_{k}}^{\mathrm{bqcf}}=\eta_{T_{k}}^{\mathrm{sac}}, \quad k \in \mathscr{K}_{\mathscr{T}_{h}}^{\mathrm{c}},
$$

where $\eta_{T_{k}}^{\mathrm{sac}}$ is defined in (3.3a), and

$$
\begin{aligned}
& \eta_{T_{\underline{L}-1}}^{\mathrm{bqcf}}=\left\{\frac{1}{2} \varepsilon\left[\sum_{\ell=\ell_{\underline{L}-2}-2}^{\ell_{\underline{L}-1}+3}\left(R_{\ell}^{\mathrm{int}}\right)^{2}\right]+\varepsilon\left[\sum_{\ell=\ell_{\underline{L}-1}-2}^{\ell_{\underline{L}-1}}\left(R_{\ell}^{\mathrm{int}}\right)^{2}\right]\right. \\
&\left.+2\left[\varepsilon \sum_{\ell \in \mathscr{B}^{l}}\left(R_{\ell}^{\mathrm{int}} \beta_{\ell}\right)^{2}\right]+2\left[\varepsilon\left|\mathscr{B}^{r}\right| \sum_{\ell \in \mathscr{B}^{l}}|\ell-1|\left|\varepsilon R_{\ell} \beta_{\ell}^{\prime}\right|^{2}\right]\right\}^{\frac{1}{2}}, \\
& \eta_{T_{\bar{L}+2}^{\mathrm{bqcf}}=}=\left\{\varepsilon\left[\sum_{\ell=\ell_{\bar{L}+1}}^{\ell_{\bar{L}}+3}\left(R_{\ell}^{\mathrm{int}}\right)^{2}\right]+\frac{1}{2} \varepsilon\left[\sum_{\ell=\ell_{\bar{L}+2}-2}^{\ell_{\bar{L}^{+2}}+3}\left(R_{\ell}^{\mathrm{int}}\right)^{2}\right]\right. \\
&\left.+2\left[\varepsilon \sum_{\ell \in \mathscr{B}^{r}}\left(R_{\ell}^{\mathrm{int}} \beta_{\ell}\right)^{2}\right]+2\left[\varepsilon\left|\mathscr{B}^{r}\right| \sum_{\ell \in \mathscr{B}^{r}}|\ell-1|\left|\varepsilon R_{\ell} \beta_{\ell}^{\prime}\right|^{2}\right]\right\}^{\frac{1}{2}},
\end{aligned}
$$

where $R_{\ell}^{\text {int }}$ is defined in (3.3b) and the sets $\mathscr{B}^{l}$ and $\mathscr{B}^{r}$ referring to the left and right blending regions are defined by

$$
\mathscr{B}^{l}:=\left\{\ell_{\underline{L}}, \cdots, \ell_{\underline{K}}\right\} \quad \text { and } \quad \mathscr{B}^{r}:=\left\{\ell_{\bar{K}}, \cdots, \ell_{\bar{L}}-1, \ell_{\bar{L}}\right\},
$$

and $|\mathscr{D}|:=\operatorname{card}(\mathscr{D})$ denotes the cardinality for a set $\mathscr{D}$.

Proof. By the variational formulation of the atomistic model (2.8) and the BQCF model (2.25), we have

$$
\begin{aligned}
R_{\mathrm{int}}^{\mathrm{bacf}}[v] & =\varepsilon \sum_{\ell \in \mathscr{L}} \sigma_{\ell}^{\mathrm{a}}\left(y_{\mathrm{ac}}\right)(v)_{\ell}^{\prime}-\varepsilon \sum_{\ell \in \mathscr{B} \cup \mathscr{C}} \sigma_{\ell}^{\mathrm{c}}\left(y_{\mathrm{ac}}\right)\left(v_{\mathrm{c}}\right)_{\ell}^{\prime}-\varepsilon \sum_{\ell \in \mathscr{L}} \sigma_{\ell}^{\mathrm{a}}\left(y_{\mathrm{ac}}\right)\left(v_{\mathrm{a}}\right)_{\ell}^{\prime} \\
& =\varepsilon \sum_{\ell \in \mathscr{C}}\left[\sigma_{\ell}^{\mathrm{c}}\left(y_{\mathrm{ac}}\right)-\sigma_{\ell}^{\mathrm{a}}\left(y_{\mathrm{ac}}\right)\right] v_{\ell}^{\prime}+\varepsilon \sum_{\ell \in \mathscr{B}}\left[\sigma_{\ell}^{\mathrm{a}}\left(y_{\mathrm{ac}}\right) v_{\ell}^{\prime}-\sigma_{\ell}^{\mathrm{a}}\left(y_{\mathrm{ac}}\right)\left(v_{\mathrm{a}}\right)_{\ell}^{\prime}-\sigma_{\ell}^{\mathrm{c}}\left(y_{\mathrm{ac}}\right)\left(v_{\mathrm{c}}\right)_{\ell}^{\prime}\right] \\
& =: R_{1}^{\mathrm{bqcf}}[v]+R_{2}^{\mathrm{bqcf}}[v] .
\end{aligned}
$$

The analysis for $R_{1}^{\mathrm{bqcf}}$ is the same as the SAC method and we concentrate on $R_{2}^{\mathrm{bqcf}}$ in (3.9). 
By the definition of $v_{\mathrm{a}}$ and $v_{\mathrm{c}}$, it is easy to verify that $v_{\ell}^{\prime}=\left(v_{\mathrm{a}}\right)_{\ell}^{\prime}+\left(v_{\mathrm{c}}\right)_{\ell}^{\prime}$ which results in

$$
\begin{aligned}
R_{2}^{\mathrm{bqcf}}[v] & =\varepsilon \sum_{\ell \in \mathscr{B}}\left[\sigma_{\ell}^{\mathrm{a}}\left(y_{\mathrm{ac}}\right)-\sigma_{\ell}^{\mathrm{c}}\left(y_{\mathrm{ac}}\right)\right]\left(v_{\mathrm{c}}\right)_{\ell}^{\prime}=\sum_{\ell \in \mathscr{B}} \varepsilon R_{\ell}^{\mathrm{int}}(\beta v)_{\ell}^{\prime} \\
& =\varepsilon \sum_{\ell \in \mathscr{B}} R_{\ell}^{\mathrm{int}} \beta_{\ell}^{\prime} v_{\ell-1}+\varepsilon \sum_{\ell \in \mathscr{B}} R_{\ell}^{\mathrm{int}} \beta_{\ell} v_{\ell}^{\prime} .
\end{aligned}
$$

For simplicity, we only present the analysis on the right blending region.

For the first part on the right hand side of (3.10), the key observation is that, by the condition that $v_{0}=0$,

$$
\begin{aligned}
\left|v_{\ell-1}\right| & =\left|\varepsilon\left(\frac{v_{\ell-1}-v_{\ell-2}}{\varepsilon}+\cdots+\frac{v_{\ell}-v_{0}}{\varepsilon}\right)\right| \\
& =\varepsilon\left|\sum_{j=1}^{\ell-1} v_{j}^{\prime}\right| \leq \varepsilon|\ell-1|^{\frac{1}{2}}\left(\sum_{j=1}^{\ell-1}\left|v_{j}^{\prime}\right|^{2}\right)^{\frac{1}{2}},
\end{aligned}
$$

where the last inequality holds by the Cauchy-Schwarz inequality. A further application of the Cauchy-Schwarz inequality yields

$$
\begin{aligned}
\varepsilon \sum_{\ell \in \mathscr{B}^{r}} R_{\ell}^{\mathrm{int}} \beta_{\ell}^{\prime} v_{\ell-1} & \leq \varepsilon \sum_{\ell \in \mathscr{B}^{r}}\left|\varepsilon R_{\ell} \beta_{\ell}^{\prime}\right||\ell-1|^{\frac{1}{2}}\left(\sum_{j=1}^{\ell-1}\left|v_{j}^{\prime}\right|^{2}\right)^{\frac{1}{2}} \\
& \leq\left[\varepsilon \sum_{\ell \in \mathscr{B}^{r}}|\ell-1|\left|\varepsilon R_{\ell} \beta_{\ell}^{\prime}\right|^{2}\right]^{\frac{1}{2}}\left[\varepsilon \sum_{\ell \in \mathscr{B}^{r}} \sum_{j=1}^{\ell-1}\left|v_{j}^{\prime}\right|^{2}\right]^{\frac{1}{2}} \\
& \leq\left[\varepsilon\left|\mathscr{B}^{r}\right| \sum_{\ell \in \mathscr{B}^{r}}|\ell-1|\left|\varepsilon R_{\ell} \beta_{\ell}^{\prime}\right|^{2}\right]^{\frac{1}{2}}\left\|v^{\prime}\right\|_{\ell_{\varepsilon}^{2}\left(\widetilde{\mathscr{B}^{r}}\right)}
\end{aligned}
$$

where $\widetilde{\mathscr{B}}{ }^{r}:=\left\{1, \cdots, \ell_{\bar{L}}\right\}$.

The same analysis can be carried out for the left blending region easily by symmetry. Together with the estimate of the second part on the right hand side of (3.10) by the Cauchy-Schwarz inequality, the following estimate holds

$$
\begin{aligned}
R_{2}^{\mathrm{bqcf}}[v] \leq & \left\{\left[\varepsilon \sum_{\ell \in \mathscr{B}^{r}}\left(R_{\ell}^{\mathrm{int}} \beta_{\ell}\right)^{2}\right]^{\frac{1}{2}}+\left[\varepsilon\left|\mathscr{B}^{r}\right| \sum_{\ell \in \mathscr{B}^{r}}|\ell-1|\left|\varepsilon R_{\ell} \beta_{\ell}^{\prime}\right|^{2}\right]^{\frac{1}{2}}\right\}\left\|v^{\prime}\right\|_{\ell_{\varepsilon}^{2}\left(\widetilde{\mathscr{B}^{r}}\right)} \\
& +\left\{\left[\varepsilon \sum_{\ell \in \mathscr{B}^{l}}\left(R_{\ell}^{\mathrm{int}} \beta_{\ell}\right)^{2}\right]^{\frac{1}{2}}+\left[\varepsilon\left|\mathscr{B}^{l}\right| \sum_{\ell \in \mathscr{B}^{l}}|\ell-1|\left|\varepsilon R_{\ell} \beta_{\ell}^{\prime}\right|^{2}\right]^{\frac{1}{2}}\right\}\left\|v^{\prime}\right\|_{\ell_{\varepsilon}^{2}\left(\widetilde{\mathscr{B}^{l}}\right)} \\
\leq & \left\{2\left[\varepsilon \sum_{\ell \in \mathscr{B}^{r}}\left(R_{\ell}^{\mathrm{int}} \beta_{\ell}\right)^{2}\right]+2\left[\varepsilon\left|\mathscr{B}^{r}\right| \sum_{\ell \in \mathscr{B}^{r}}|\ell-1|\left|\varepsilon R_{\ell} \beta_{\ell}^{\prime}\right|^{2}\right]\right. \\
& \left.+2\left[\varepsilon \sum_{\ell \in \mathscr{B}^{l}}\left(R_{\ell}^{\mathrm{int}} \beta_{\ell}\right)^{2}\right]+2\left[\varepsilon\left|\mathscr{B}^{l}\right| \sum_{\ell \in \mathscr{B}^{l}}|\ell-1|\left|\varepsilon R_{\ell} \beta_{\ell}^{\prime}\right|^{2}\right]\right\}^{\frac{1}{2}}\left\|v^{\prime}\right\|_{\ell_{\varepsilon}^{2}(\widetilde{\mathscr{B}})}
\end{aligned}
$$


where $\widetilde{\mathscr{B}^{l}}:=\{\underline{L}, \cdots, 0\}$ and $\widetilde{\mathscr{B}}:=\{\underline{L}, \cdots, \bar{L}\}$, and the last inequality is obtained by a weighted Cauchy-Schwarz inequality. The stated results can be obtained after the combination of $R_{1}^{\text {bqcf }}$ and the estimate of $R_{2}^{\text {bqcf }}$ in (3.13) with the application of the Cauchy-Schwarz inequality.

For the reason we mentioned in Remark 3.1, we present a nodewise internal residual estimate in the following corollary for the case $\mathscr{U}^{h} \equiv \mathscr{U}^{\varepsilon}$ and $\mathscr{Y}^{h} \equiv \mathscr{Y}^{\varepsilon}$.

Corollary 3.2 (Nodewise internal residual estimate for BQCF). Let $y_{\mathrm{ac}}$ be a solution of the BQCF model (2.24) with the solution spaces $\mathscr{U}^{h} \equiv \mathscr{U}^{\varepsilon}$ and $\mathscr{Y}^{h} \equiv \mathscr{Y}^{\varepsilon}$. With the same assumption of $y_{\mathrm{ac}}$ and the same definition of $R_{\mathrm{int}}^{\mathrm{sac}}$ in Theorem 3.2, the internal residual is estimated by

$$
\left\|R_{\text {int }}^{\mathrm{bqcf}}\right\|_{\mathscr{U}^{-1,2}} \leq\left\{\sum_{\ell \in \mathscr{L}_{\ell}^{\mathrm{c}}}\left(\tilde{\eta}_{\ell}^{\mathrm{bqcf}}\right)^{2}\right\}^{\frac{1}{2}}:=\eta_{\text {int }}^{\mathrm{bqcf}},
$$

where the nodewise upper bound of the internal residual is given by

$$
\begin{aligned}
& \left(\tilde{\eta}_{\ell}^{\mathrm{bqcf}}\right)^{2}:=\frac{1}{5} \sum_{\ell-2}^{\ell+2}\left(\eta_{\ell}^{\mathrm{bqcf}}\right)^{2}, \\
& \eta_{\ell}^{\mathrm{bqcf}}= \begin{cases}\varepsilon R_{\ell}^{\mathrm{int}}, & \ell \in \mathscr{C}, \\
\left.\left\{2\left[\varepsilon\left(R_{\ell}^{\mathrm{int}} \beta_{\ell}\right)^{2}\right]+2 \varepsilon\left|\mathscr{B}^{l}\right||\ell-1|\left|\varepsilon R_{\ell} \beta_{\ell}^{\prime}\right|^{2}\right]\right\}^{\frac{1}{2}}, & l=\mathscr{B}^{l}, \\
\left.\left\{2\left[\varepsilon\left(R_{\ell}^{\mathrm{int}} \beta_{\ell}\right)^{2}\right]+2 \varepsilon\left|\mathscr{B}^{r}\right||\ell-1|\left|\varepsilon R_{\ell} \beta_{\ell}^{\prime}\right|^{2}\right]\right\}^{\frac{1}{2}}, & l=\mathscr{B}^{r} .\end{cases}
\end{aligned}
$$

Remark 3.2. As we mentioned in Remark 2.2, we may consider QCF as a special case of BQCF where we simply define $\mathscr{B}:=\left\{\ell_{\underline{K}}-1, \ell_{\underline{K}}\right\} \cup\left\{\ell_{\bar{K}}, \ell_{\bar{K}}+1\right\}:=\mathscr{B}^{l} \cup \mathscr{B}^{r}$ and the internal residual can be obtained accordingly.

\subsection{Estimate of the external residual}

We then turn to the estimate of the residual of the external energy defined by

$$
R_{\mathrm{ext}}[v]=\left\langle g, I_{h} v\right\rangle_{h}-\langle g, v\rangle_{\varepsilon},
$$

where $I_{h} v$ is the nodal interpolant of $v$ with respect to $\mathscr{T}_{h}$. We essentially follow the same line as in $[32,34]$ for the estimate of $R_{\text {ext }}$ in which the analysis is given in detail. We decide to include a brief discussion but not just citing the related work simply for the thoroughness of the paper which also provides the rationale for the numerical experiments in Section 6.

The starting point is to transform (3.16) to a continuous problem by treating the test function $v$ as an element in $\mathscr{P}_{1}\left(\mathscr{T}_{\varepsilon}\right)$ and $g$ as a $C^{2}$ function on $\Omega_{c}$. By such consideration 
we may separate $R_{\text {ext }}$ by

$$
\begin{aligned}
R_{\text {ext }}[v] & =\int_{\Omega_{\mathrm{c}}}\left[I_{\varepsilon}(g v)-I_{h}\left(g I_{h} v\right)\right] \mathrm{d} x \\
& =\int_{\Omega_{\mathrm{c}}}\left[I_{\varepsilon}(g v)-g v\right] \mathrm{d} x+\int_{\Omega_{\mathrm{c}}}\left[g v-g I_{h} v\right] \mathrm{d} x+\int_{\Omega_{\mathrm{c}}}\left[g I_{h} v-I_{h}\left(g I_{h} v\right)\right] \mathrm{d} x \\
& =: R_{\text {ext }}^{1}[v]+R_{\text {ext }}^{2}[v]+R_{\text {ext }}^{3}[v],
\end{aligned}
$$

where $R_{\text {ext }}^{2}[v]$ represents the residual by the coarse-graining of the solution space and $R_{\text {ext }}^{1}[v]$ and $R_{\text {ext }}^{3}[v]$ represents the residual by the application of quadrature rules. By standard interpolation error estimates and Poincaré inequality for $H^{1}(\Omega)$, we obtain (c.f. [34, Proposition 3])

$$
\begin{aligned}
R_{\mathrm{ext}}^{1}[v] \leq & \eta^{f}\left\|v^{\prime}\right\|_{L^{2}(\Omega)}:=\left[\sum_{k \in K_{\mathscr{T}_{h}}^{c}}\left(\frac{h_{k}^{2}}{\pi^{2}}\|g\|_{L^{2}\left(T_{k}^{h}\right)}^{2}\right)\right]^{\frac{1}{2}}\left\|v^{\prime}\right\|_{L^{2}(\Omega)} \\
= & :\left[\sum_{k \in K_{\mathscr{T}_{h}}^{\mathrm{c}}} \eta_{T_{k}}^{f}\right]^{\frac{1}{2}}\left\|v^{\prime}\right\|_{L^{2}(\Omega)}, \\
R_{\mathrm{ext}}^{2}[v]+ & R_{\mathrm{ext}}^{3}[v] \leq \eta^{q}\left\|v^{\prime}\right\|_{L^{2}(\Omega)} \\
= & :\left[\sum_{k \in K_{\mathscr{T}_{h}}^{c}}\left(\left(\varepsilon^{4}+h_{k}^{4}\right)\left\|g^{\prime}\right\|_{L^{2}\left(T_{k}^{h}\right)}^{2}+\frac{\left(\varepsilon^{4}+h_{k}^{4}\right)}{4 \pi^{2}}\left\|g^{\prime \prime}\right\|_{L^{2}\left(T_{k}^{h}\right)}^{2}\right)\right]^{\frac{1}{2}}\left\|v^{\prime}\right\|_{L^{2}(\Omega)} .
\end{aligned}
$$

We observe that the estimate of the quadrature residual $\eta^{q}$ is of higher order of the estimate of the main residual $\eta^{f}$. However, such assertion is inadequate if we consider the singular force which essentially behaves like $|g(x)| \sim|x|^{-1}$ near $x=0$ (which is the force we employ in our numerical experiment). In that case, one can show that the reverse is true, i.e., $\eta^{f}$ is dominated by $\eta^{q}$ because of the large value of $\left\|g^{\prime \prime}\right\|_{L^{2}\left(T_{k}^{h}\right)}$ near $x=0$.

The remedy comes from the application of a weighted Poincaré inequality. We choose a proper weight function $w$ such that $\left\|w g^{\prime \prime}\right\|_{L^{2}(\Omega)}$ is small enough but $\left\|w^{-1} v\right\|_{L^{2}(\Omega)} \leq$ $C\left\|v^{\prime}\right\|_{L^{2}(\Omega)}$ for some $C$ not too large. The estimate of $R_{\text {ext }}^{3}$ then becomes

$$
\begin{aligned}
& \left|R_{\text {ext }}^{3}[v]\right| \leq \frac{1}{4}\left\|h^{2}\left(g I_{h} v\right)^{\prime \prime}\right\|_{L^{1}\left(\Omega_{\mathrm{c}}\right)} \leq \frac{1}{2}\left\|h^{2} g^{\prime} I_{h} v^{\prime}\right\|_{L^{1}\left(\tilde{\Omega}_{\mathrm{c}}\right)}+\frac{1}{4}\left\|h^{2} g^{\prime \prime} I_{h} v\right\|_{L^{1}\left(\tilde{\Omega}_{\mathrm{c}}\right)} \\
= & \frac{1}{2}\left\|h^{2} g^{\prime} I_{h} v^{\prime}\right\|_{L^{1}\left(\Omega_{\mathrm{c}}\right)}+\frac{1}{4}\left\|\left(h^{2} w g^{\prime \prime}\right)\left(w^{-1} I_{h} v\right)\right\|_{L^{1}\left(\Omega_{\mathrm{c}}\right)} \\
\leq & \frac{1}{2}\left\|h^{2} g^{\prime}\right\|_{L^{2}\left(\Omega_{\mathrm{c}}\right)}\left\|v^{\prime}\right\|_{L^{2}\left(\Omega_{\mathrm{c}}\right)}+\frac{1}{4} C\left\|h^{2} w g^{\prime \prime}\right\|_{L^{2}\left(\Omega_{\mathrm{c}}\right)}\left\|v^{\prime}\right\|_{L^{2}} .
\end{aligned}
$$

We consequently have (the estimate of $R_{\text {ext }}^{2}$ is essentially the same)

$$
\left\|R_{\text {ext }}\right\|_{\mathscr{U}^{-1,2}} \leq \eta^{f}+\hat{\eta}^{q}:=\eta_{\mathrm{ext}}
$$


where

$$
\begin{aligned}
& \left(\hat{\eta}^{q}\right)^{2}:=\sum_{\substack{k \in\{1, \cdots, K\} \\
T_{k}^{h} \subset \tilde{\Omega}_{c}}}\left(\hat{\eta}_{k}^{q}\right)^{2}, \\
& \left(\hat{\eta}_{k}^{q}\right)^{2}:=\left(\varepsilon^{4}+h_{k}^{4}\right)\left\|g^{\prime}\right\|_{L^{2}\left(T_{k}^{h}\right)}^{2}+\left(\varepsilon^{4}+h_{k}^{4}\right) C\left\|w g^{\prime \prime}\right\|_{L^{2}\left(T_{k}^{h}\right)^{h}}^{2} .
\end{aligned}
$$

For the singular force in our numerical experiment, we choose $w(x):=x \log (x)$ and consequently $C=\frac{1}{\log 2}$. We can then prove $\hat{\eta}^{q}$ is dominated by $\eta^{f}$. For the technical detail we refer to [34, Section 3.3 and 3.4].

\section{Stability}

In order to give the a posteriori error estimates for the QC methods, we need the corresponding a posteriori stability condition. The correct notion of such condition is the positive definiteness (coercivity) of the Hessian of the atomistic model at the QC solutions as we aim to derive the error bounds in $\mathscr{U}^{1,2}$-norm. The following discussion for stability can also be deemed as an extension of that in [34] for multi-body interaction potentials.

We first define the set of interaction range of an atom to be

$$
\mathscr{R}=\{1,-1,2,-2\},
$$

and define the sets of the nearest and the next-nearest neighbour interaction ranges as

$$
\mathscr{R}^{\mathrm{NN}}=\{1,-1\}, \quad \mathscr{R}^{\mathrm{NNN}}=\{2,-2\} .
$$

By direct calculation we obtain the second variation of the atomistic energy at $y \in \mathscr{Y}^{\varepsilon}$ by

$$
\begin{aligned}
& \left\langle\delta^{2} \mathscr{E}^{\mathrm{a}}(y) v, v\right\rangle \\
& =\varepsilon \sum_{\ell \in \mathscr{L}} \sum_{\rho \in \mathscr{R}} \partial_{\rho, \rho} V\left(D y_{\ell}\right)\left(D_{\rho} v_{\ell}\right)^{2}+\varepsilon \sum_{\ell \in \mathscr{L}} \sum_{\substack{\rho, \xi \in \mathscr{R}^{\mathrm{NN}} \\
\rho \neq \xi}} \partial_{\rho, \xi} V\left(D y_{\ell}\right)\left(D_{\rho} v_{\ell}\right)\left(D_{\xi} v_{\ell}\right) \\
& +\varepsilon \sum_{\ell \in \mathscr{L}} \sum_{\substack{\rho \in \mathscr{R}^{\mathrm{NN}}, \xi \in \mathscr{R}^{\mathrm{NNN}} \\
\text { or } \xi \in \mathscr{R}^{\mathrm{NN}}, \rho \in \mathscr{R}}} \partial_{\rho, \xi} V\left(D y_{\ell}\right)\left(D_{\rho} v_{\ell}\right)\left(D_{\xi} v_{\ell}\right) \\
& +\varepsilon \sum_{\ell \in \mathscr{L}} \sum_{\substack{\rho, \xi \in \mathscr{R} \\
\rho \neq \xi}} \partial_{\rho, \xi} V\left(D y_{\ell}\right)\left(D_{\rho} v_{\ell}\right)\left(D_{\xi} v_{\ell}\right) \\
& =: \operatorname{Stab}_{1}+\mathrm{Stab}_{2}+\mathrm{Stab}_{3}+\mathrm{Stab}_{4} \text {. }
\end{aligned}
$$

By the inequality of arithmetic mean and and the number of combinations in each case, 
we can estimate the last three parts of (4.3) by

$$
\begin{aligned}
& \mathrm{Stab}_{2} \geq \sum_{\ell \in \mathscr{L}} C_{\ell}^{\mathrm{cross}, \mathrm{NN}} \sum_{\rho \in \mathscr{R} \mathrm{NN}}\left(D_{\rho} v_{\ell}\right)^{2}, \\
& \mathrm{Stab}_{3} \geq \sum_{\ell \in \mathscr{L}} C_{\ell}^{\mathrm{cross}, \mathrm{NN}-\mathrm{NNN}} \sum_{\rho \in \mathscr{R}}\left(D_{\rho} v_{\ell}\right)^{2}, \\
& \mathrm{Stab}_{4} \geq \sum_{\ell \in \mathscr{L}} C_{\ell}^{\mathrm{cross}, \mathrm{NNN}} \sum_{\rho \in \mathscr{R} \mathrm{NNN}},\left(D_{\rho} v_{\ell}\right)^{2},
\end{aligned}
$$

where

$$
\begin{aligned}
& C_{\ell}^{\text {cross,NN }}=\min \left\{0, \min _{\substack{\rho, \xi \in \mathscr{R}^{\mathrm{NN}} \\
\xi \neq \rho}} \partial_{\rho, \xi} V\left(D y_{\ell}\right)\right\}, \\
& C_{\ell}^{\text {cross,NN-NNN }}=\left\{0, \min _{\substack{\rho \in \mathscr{R}^{\mathrm{NN}}, \xi \in \mathscr{R} \mathrm{NNN} \\
\text { or } \xi \in \mathscr{R}^{\mathrm{NN}}, \rho \in \mathscr{R}^{\mathrm{NNN}}}} \partial_{\rho, \xi} V\left(D y_{\ell}\right)\right\}, \\
& C_{\ell}^{\text {cross,NNN }}=\min \left\{0, \min _{\substack{\rho, \xi \in \mathscr{R} \text { NNN } \\
\xi \neq \rho}} \partial_{\rho, \xi} V\left(D y_{\ell}\right)\right\} .
\end{aligned}
$$

Defining

$$
C_{\ell}^{\mathrm{NN}}=\min _{\rho \in \mathscr{R}^{\mathrm{NN}}} \partial_{\rho, \rho} V\left(D y_{\ell}\right), \quad C_{\ell}^{\mathrm{NNN}}=\min _{\rho \in \mathscr{R}^{\mathrm{NNN}}} \partial_{\rho, \rho} V\left(D y_{\ell}\right),
$$

we can separate the terms involving the nearest neighbour interactions and the nextnearest neighbour interactions and estimate (4.3) by

$$
\begin{aligned}
& \left\langle\delta^{2} \mathscr{E}^{\mathrm{a}}(y) v, v\right\rangle \\
\geq & \varepsilon \sum_{\ell \in \mathscr{L}} \sum_{\rho \in \mathscr{R}^{N N}}\left[C_{\ell}^{\mathrm{NN}}+C_{\ell}^{\mathrm{cross}, \mathrm{NN}}+C_{\ell}^{\mathrm{cross}, \mathrm{NN}-\mathrm{NNN}}\right]\left(D_{\rho} v_{\ell}\right)^{2} \\
& +\varepsilon \sum_{\ell \in \mathscr{L}} \sum_{\rho \in \mathscr{R}^{N N N}}\left[C_{\ell}^{\mathrm{NNN}}+C_{\ell}^{\mathrm{cross}, \mathrm{NNN}}+C_{\ell}^{\mathrm{cross}, \mathrm{NN}-\mathrm{NNN}}\right]\left(D_{\rho} v_{\ell}\right)^{2} \\
= & \varepsilon \sum_{\ell \in \mathscr{L}} C_{\ell}^{1}\left[\left|v_{\ell}^{\prime}\right|^{2}+\left|v_{\ell+1}^{\prime}\right|^{2}\right]+\varepsilon \sum_{\ell \in \mathscr{L}} C_{\ell}^{2}\left[\left|v_{\ell}^{\prime}+v_{\ell-1}^{\prime}\right|^{2}+\left|v_{\ell+1}^{\prime}+v_{\ell+2}^{\prime}\right|^{2}\right],
\end{aligned}
$$

where

$$
C_{\ell}^{1}=C_{\ell}^{\mathrm{NN}}+C_{\ell}^{\mathrm{cross}, \mathrm{NN}}+C_{\ell}^{\mathrm{cross}, \mathrm{NN}-\mathrm{NNN}}, \quad C_{\ell}^{2}=C_{\ell}^{\mathrm{NNN}}+C_{\ell}^{\mathrm{cross}, \mathrm{NNN}}+C_{\ell}^{\mathrm{cross}, \mathrm{NN}-\mathrm{NNN}} .
$$

The technique for estimating (4.7) is that we rewrite the 'non-local' Hessian terms $\mid v_{\ell}^{\prime}+$ $\left.v_{\ell+1}^{\prime}\right|^{2}$ in terms of the 'local' terms $\left|v_{\ell}^{\prime}\right|^{2}$ and $\left|v_{\ell+1}^{\prime}\right|^{2}$ and a strain-gradient correction $[31,34]$,

$$
\left|v_{\ell}^{\prime}+v_{\ell+1}^{\prime}\right|^{2}=2\left|v_{\ell}^{\prime}\right|^{2}+2\left|v_{\ell+1}^{\prime}\right|^{2}-\varepsilon^{2}\left|v_{\ell}^{\prime \prime}\right|^{2},
$$

which yields the following estimate

$$
\left\langle\delta^{2} \mathscr{E}^{\mathrm{a}}(y) v, v\right\rangle \geq \varepsilon \sum_{\ell \in \mathscr{L}} A_{\ell}(y)\left|v_{\ell}^{\prime}\right|^{2}+\varepsilon^{3} \sum_{\ell \in \mathscr{L}} B_{\ell}(y)\left|v_{\ell}^{\prime \prime}\right|^{2},
$$


where

$$
\begin{aligned}
& A_{\ell}=C_{\ell}^{1}+C_{\ell-1}^{1}+2 C_{\ell-2}^{2}+2 C_{\ell-1}^{2}+2 C_{\ell}^{2}+2 C_{\ell+1}^{2}, \\
& B_{\ell}=-C_{\ell-1}^{2}-C_{\ell+1}^{2} .
\end{aligned}
$$

The same as for pair potential [34], we assume that $B_{\ell} \geq 0$, which will also be verified numerically in Section 6.2.2. Consequently we obtain the following lemma which gives the sufficient conditions for the stability of the atomistic hessian evaluated at the QC solution.

Proposition 4.1. Let $y_{\mathrm{ac}} \in \mathscr{Y}^{h}$, ac $\in\{\mathrm{qcf}$, sac, bqcf $\}$ be a solution of the force-based coupling problems (2.20), (2.22) and (2.24) respectively and assume that $B_{\ell}\left(y_{\text {ac }}\right) \geq 0, \forall \ell \in \mathscr{L}$ where the coefficients $B_{\ell}(y)$ are defined in (4.9a); then

$$
\left\langle\delta^{2} \mathscr{E}^{\mathrm{a}}\left(y_{\mathrm{ac}}\right) v, v\right\rangle \geq A_{*}\left(y_{\mathrm{ac}}\right)\left\|v^{\prime}\right\|_{\ell_{\varepsilon}^{2}}^{2}, \quad \forall v \in \mathscr{U}^{\varepsilon}, \quad \text { where } \quad A_{*}\left(y_{\mathrm{ac}}\right):=\min _{\ell \in \mathscr{L}} A_{\ell}\left(y_{\mathrm{ac}}\right),
$$

and the $A_{\ell}(y)$ is defined in (4.9a).

Remark 4.1. We briefly comment on the stability estimate given. First of all, We emphasize that the computational cost of $A_{*}\left(y_{\mathrm{ac}}\right)$ is of $\mathscr{O}(K)$ since the deformation gradient remains constant in each element. Second, We treat the nearest neighbour cross terms separately to single out its importance compared with other cross terms which will be illustrated in our numerical examples. Third, the sharpness of the stability estimate may be improved with some extra effort but we decide not to include it in this work but will show that the current stability estimate is sufficient for the numerical experiments we consider in Section 6. Finally, for pair interaction potentials, $B_{\ell}<0$ may only be achieved under extreme compression under which case the pair potentials may not be considered correct. We thus expect similar facts holds for multi-body potentials we consider.

\subsection{Estimates for the Hessian}

Before we present our main theorems, we state a useful auxiliary results, a local Lipschitz bound on $\delta^{2} \mathscr{E}^{\text {a }}$, whose proofs is given in Appendix A.

Lemma 4.1. Let $y, z \in \mathscr{Y}^{\varepsilon}$ such that $y_{\ell}, z_{\ell} \in E:=[\mu, v]$ for some constants $\mu<v$, then

$$
\left|\left\langle\left\{\delta^{2} \mathscr{E}^{\mathrm{a}}(y)-\delta^{2} \mathscr{E}^{\mathrm{a}}(z)\right\} v, w\right\rangle\right| \leq C_{\mathrm{Lip}}\left\|y^{\prime}-z^{\prime}\right\|_{\ell_{\varepsilon}^{\infty}}\left\|v^{\prime}\right\|_{\ell_{\varepsilon}^{2}}\left\|w^{\prime}\right\|_{\ell_{\varepsilon}^{2}} \quad \forall v, w \in \mathscr{U},
$$

where

$$
C_{\text {Lip }}=2 \bar{M}_{2}(E)+22 \underline{M}_{2}(E)+72 \bar{m}_{2}(E)+120 \underline{m}_{2}(E) .
$$

Here, $\underline{m}_{j}(E), \bar{m}_{j}(E), \underline{M}_{j}(E)$ and $\bar{M}_{j}(E)$ are defined in Appendix A. 


\section{A Posteriori error estimates}

We now arrive at the point to present the complete a posteriori error estimate by combining the residual estimate and the stability estimate under certain assumptions which will be clear in the following theorem.

Theorem 5.1. Let $y_{\mathrm{ac}} \in \mathscr{Y}^{h}$, ac $\in\{\mathrm{qcf}$, sac, bqcf $\}$ be a solution of the force-based coupling problems (2.20), (2.22) and (2.24)respectively with $B_{\ell}\left(y_{\mathrm{ac}}\right) \geq 0, \forall \ell \in \mathscr{L}$ and $A_{*}\left(y_{\mathrm{ac}}\right)>0$ where $B_{\ell}$ and $A_{*}$ are defined in (4.9a) and (4.10). Suppose $y_{\mathrm{a}}$ is a solution of the atomistic problem (2.4) such that, for some $\tau>0$,

$$
\left\|y_{\mathrm{a}}^{\prime}-y_{\mathrm{qc}}^{\prime}\right\|_{\ell_{\varepsilon}^{\infty}} \leq \tau .
$$

If $\tau$ is sufficiently small, then we have the error estimate

$$
\left\|y_{\mathrm{a}}^{\prime}-y_{\mathrm{ac}}^{\prime}\right\|_{\ell_{\varepsilon}^{2}} \leq \frac{2}{A_{*}\left(y_{\mathrm{qc}}\right)}\left(\eta_{\mathrm{int}}^{\mathrm{ac}}+\eta_{\mathrm{ext}}\right)
$$

where $\eta_{\mathrm{int}}^{\mathrm{ac}}$ are defined in (3.2) and (3.6) and $\eta^{\mathrm{ext}}$ is defined in (3.20).

Proof. Let $e:=y_{\mathrm{a}}-y_{\mathrm{ac}} \in \mathscr{U}^{\varepsilon}$. By the mean value theorem we know that there exists $\theta \in \operatorname{conv}\left\{y_{\mathrm{a}}, y_{\mathrm{ac}}\right\}$ such that

$$
\left|\left\langle\delta^{2} \mathscr{E}^{a}(\theta) e, e\right\rangle\right|=\left|\left\langle\delta \mathscr{E}^{a}\left(y_{\mathrm{ac}}\right), e\right\rangle-\left\langle\delta \mathscr{E}^{a}\left(y_{\mathrm{a}}\right), e\right\rangle\right|=\left|\left\langle\delta \mathscr{E}^{a}\left(y_{\mathrm{ac}}\right), e\right\rangle-\langle g, e\rangle_{\varepsilon}\right|,
$$

where the right hand side of the above equation is exactly the residual $R[v]$ defined in (3.1) and we therefore have

$$
\left|\left\langle\delta^{2} \mathscr{E} a(\theta) e, e\right\rangle\right|=\left|R_{\mathrm{int}}[v]+R_{\mathrm{ext}}[v]\right| \leq\left[\eta_{\mathrm{int}}^{\mathrm{ac}}+\eta_{\mathrm{ext}}\right]\left\|e^{\prime}\right\|_{\ell_{\varepsilon}^{2}}
$$

by the estimates (3.2), (3.6) and (3.20).

We then compute a lower bound on $\left|\left\langle\delta^{2} \mathscr{E}^{a}(\theta) e, e\right\rangle\right|$. Using the Lipschitz estimate given in Lemma 4.1, the stability estimate in Proposition 4.1 together with our assumption on $B_{\ell}\left(y_{\mathrm{ac}}\right)$ and $A_{*}\left(y_{\mathrm{ac}}\right)$ and the a priori bound (5.1), we have

$$
\begin{aligned}
\left|\left\langle\delta^{2} \mathscr{E}^{a}(\theta) e, e\right\rangle\right| & \geq \mid\left\langle\delta^{2} \mathscr{E}^{a}\left(y_{\mathrm{ac}}\right) e, e\right\rangle-C_{\mathrm{Lip}}\left\|\theta-y_{\mathrm{ac}}\right\|\left\|e^{\prime}\right\|_{\ell_{\varepsilon}^{2}}^{2} \\
& \geq \mid\left\langle\delta^{2} \mathscr{E}^{a}\left(y_{\mathrm{ac}}\right) e, e\right\rangle-C_{\mathrm{Lip}}\left\|y_{\mathrm{a}}-y_{\mathrm{ac}}\right\|\left\|e^{\prime}\right\|_{\ell_{\varepsilon}^{2}}^{2} \\
& \geq\left(A_{*}\left(y_{\mathrm{ac}}\right)-C_{\mathrm{Lip}} \tau\right)\left\|e^{\prime}\right\|_{\ell_{\varepsilon}^{2}}^{2} .
\end{aligned}
$$

If we require that $\tau \leq A_{*}\left(y_{\text {ac }}\right) /\left(2 C_{\text {Lip }}\right)$ (this is satisfied for $\tau$ sufficiently small), we finally obtain

$$
\frac{1}{2} A_{*}\left(y_{\mathrm{qc}}\right)\left\|e^{\prime}\right\|_{\ell_{\varepsilon}^{2}}^{2} \leq\left[\eta_{\mathrm{int}}^{\mathrm{ac}}+\eta_{\mathrm{ext}}\right]\left\|e^{\prime}\right\|_{\ell_{\varepsilon}^{2}} .
$$

Dividing through by $\left\|e^{\prime}\right\|_{\ell_{\varepsilon}^{2}}$ yields the stated estimate. 
Remark 5.1. We make an important assumptions in Theorem 5.1 that $y_{\mathrm{qc}}$ is sufficiently close to $y_{\mathrm{a}}$ which is characterized by (5.1). This assumption essentially says that given a $y_{\text {qc }}$ satisfying $A_{*}\left(y_{\mathrm{ac}}\right)>0$, there exists a $y_{\mathrm{a}}$ 'sufficiently' near by. Such assumption can be dropped by proper applications of the inverse function theorem which have some existing results, c.f., $[31,41]$. However, since the a posteriori existence is not the focus of the present work, we do not go further in this direction but to keep the assumption (5.1) to for simplicity.

\section{Numerical experiments}

In this section, we present numerical experiments to illustrate the results of our analysis. In particular, we will consider the problem of mesh adaptivity and model adaptivity with the residual based error estimator. Throughout this section, We fix our computational domain $\Omega=[-1,1]$ and $F=1.03$, which corresponds to applying a 3\% tensile stretch from the ground state to the system. We assume our atomistic system possesses EAM potential whose site energy is given by

$$
V\left(D y_{\ell}\right)=\frac{1}{2} \sum_{\rho \in \mathscr{R}}\left(\phi\left(D_{i} y_{\ell}\right)+\phi\left(-D_{j} y_{\ell}\right)\right)+\widetilde{F}\left(\sum_{\rho \in \mathscr{R}}\left(\psi\left(D_{i} y_{\ell}\right)+\psi\left(-D_{j} y_{\ell}\right)\right)\right)
$$

where $\phi(r)=e^{-2 a(r-1)}-2 e^{-a(r-1)}, \psi(r)=e^{-b r}$ and $\widetilde{F}(\rho)=c\left[\left(\rho-\rho_{0}\right)^{2}+\left(\rho-\rho_{0}\right)^{4}\right]$, with the parameter $a=4.4, b=3, c=5, \rho_{0}=2 e^{-b}$.

\subsection{Mesh adaptivity}

In the first numerical experiment, we propose an adaptive mesh refinement algorithm by the residual based error estimator, and show numerically that it achieves an optimal "convergence rate" in terms of the number of degrees of freedom.

The problem we consider here follows that in [34] which is a benchmark testing case in $1 \mathrm{D}$. We set $N=50000$ and defined the external force $g_{\ell}$ to be

$$
g_{\ell}:= \begin{cases}-0.4\left(1+\frac{1}{2 \varepsilon \ell}\right), & -25000 \leq \ell<0 \\ 0.4\left(\frac{1}{2 \varepsilon \ell}-1\right), & 0<\ell \leq 25000\end{cases}
$$

We note that $g(x)$ behaves essentially like $|x|^{-1}$, which is a typical decay rate for elastic fields generated by long-ranged defects in 2D/3D [13]. We thus test the performance and robustness of our adaptive algorithm in a setting that includes some of the features of higher dimensional problems. The constant 0.4 was chosen sufficiently large to achieve a significant deformation, but sufficiently small to ensure that there exists an elastic stable equilibrium configuration. 
The blending function for the BQCF method we choose is a cubic spline defined by (c.f. [14])

$$
\hat{\beta}(x):= \begin{cases}0, & x<0, \\ -2 x^{3}+3 x^{2}, & 0 \leq x \leq 1, \\ 1, & x>1 .\end{cases}
$$

Such choice of blending function make sure the atomistic model and the local QC model are smoothly blended in the blending region and is also optimal in the sense of maintaining the positive-definiteness of the BQCF operator near tensile limit according to the analysis in $[14-16,32]$.

We use two different strategies to decide the blending width of the BQCF method. The first strategy is to fix the blending width, i.e. $\bar{L}-\bar{K}$, to be 5,10 and 20 . The second strategy is slightly adaptive where we set the the radius of the atomistic region to be of the same as the blending width, which was shown to produce the (quasi-)optimal $H^{1}$-error $[14,15]$.

We will compute the relative errors of the strain gradient, given by:

$$
\frac{\left\|y_{\mathrm{ac}}^{\prime}-y_{\mathrm{a}}^{\prime}\right\|_{\ell_{\varepsilon}^{2}}}{\left\|u_{\mathrm{a}}^{\prime}\right\|_{\ell_{\varepsilon}^{2}}},
$$

where ac $\in\{$ qcf, sac, bqcf\}.

\subsubsection{Adaptive algorithm}

In our computations, we begin with a mesh that resolves the "defect" (i.e., it has atomistic resolution near $x=0$ ) but is coarse elsewhere. We then employ the algorithm stated below to locally refine the mesh where required and thus improve the quality of the solution.

We first define the error estimators according to which we drive the mesh refinement. The element error estimator are given by (cf. (3.3a), (3.8a) and (3.18a))

$$
\left(\rho_{T_{k}}\right)^{2}:=\frac{2}{A_{*}\left(y_{\mathrm{ac}}\right)} \cdot \begin{cases}\left(\eta_{T_{k}}^{\mathrm{ac}}\right)^{2}+\left(\eta_{T_{k}}^{f}\right)^{2}, & \text { if } k \in \mathscr{K}_{\mathscr{T}_{h}}^{\mathrm{c}}, \\ 0, & \text { otherwise, }\end{cases}
$$

where ac $\in\{$ qcf, sac, bqcf $\}$. Note that we have ignored the quadrature residual $\eta^{q}$ which was shown to be dominated by $\eta^{f}$ in Section 3.4.

The following algorithm is based on established ideas from the adaptive finite element literature $[11,29]$.

\subsubsection{Numerical results}

We summarize the results of our numerical experiments for different force-based methods.

1. In Fig. 1, we display the gradient errors for the mesh generation algorithms for QCF, SAC and BQCF with different blending width. We note that the error curves of different force-based methods are barely distinguishable which implies that these methods possesses almost the same accuracy under the current setting. 
Algorithm 6.1 A posteriori mesh adaptivity.

1. Create Add the nodes $0, \pm \varepsilon, \pm 2 \varepsilon, \cdots,(\bar{L}-1) \varepsilon$ and $(\underline{L}+1) \varepsilon$ to the mesh $(\bar{L}=\bar{K}$ for the QCF and the SAC methods). Add the nodes $\left\{x_{0}^{h}=-1, x_{K}^{h}=1\right\}$ to the mesh.

2. Solve: Compute the QC solution on the current mesh, compute the error indicators $\rho_{k}$. For $T_{k}^{h} \subset \Omega_{\mathrm{a}}$, set $\rho_{k}=0$.

3. Mark: Choose a minimal subset $\mathscr{M} \subset\{1, \cdots, K\}$ of indices such that

$$
\sum_{k \in \mathscr{M}} \rho_{k} \geq \frac{1}{2} \sum_{k=1}^{K} \rho_{k} .
$$

4. Refine: Bisect all elements $T_{k}^{h}$ with indices belonging to $\mathscr{M}$ :

a For the QCF and the SAC methods if an element that needs to be refined is adjacent to the atomistic region, merge this element into the atomistic region and create a new atomistic to continuum interface.

b For the BQCF methods if an element that needs to be refined is adjacent to the blending region, merge this element into the blending region and create a new atomistic region and blending region according to the fixed blending width strategy or the adaptive blending width strategy.

5. If the resulting mesh reaches a prescribed maximal number of degrees of freedom, stop the algorithm; otherwise, go to Step (2).

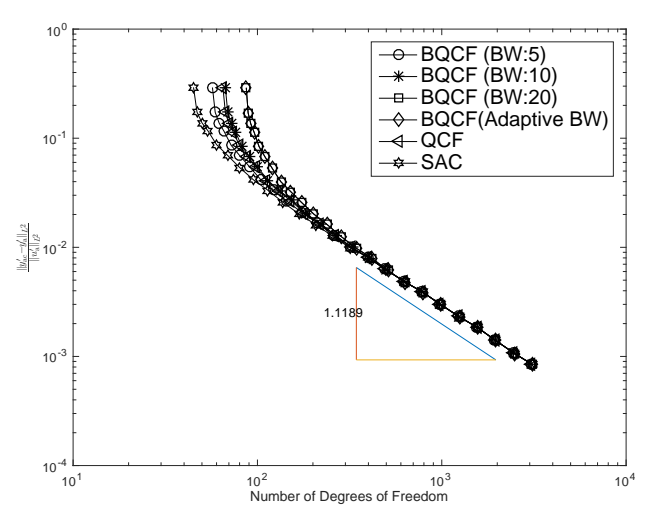

Figure 1: Relative errors in the deformation gradient plotted against the number of degrees of freedom for five types of mesh refinement.

2. We set the maximum number of degrees of freedom to be 3000 and observe the convergence rate is close to 1 due to the $\mathscr{P}_{1}$ solution space we choose.

3. We compute the efficiency factors which are the ratio between the estimated error and the true error and are shown in Fig. 2. The efficiency factors are very moderate 


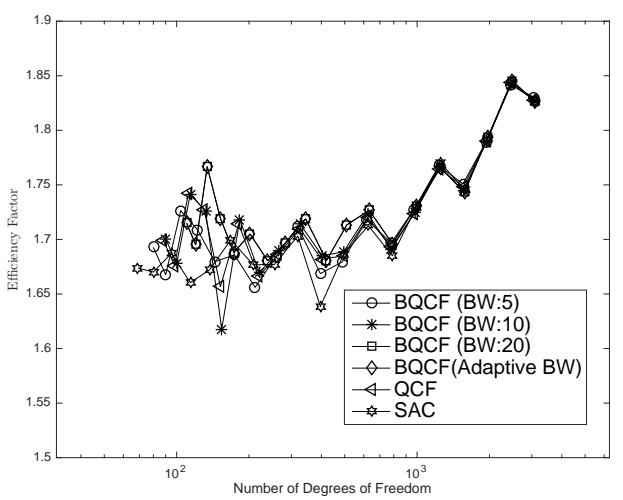

Figure 2: Efficiency factors plotted against the number of degrees of freedom for five types of mesh refinement.

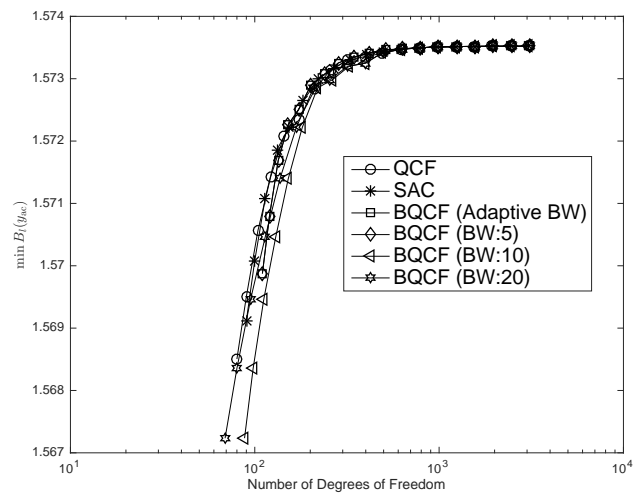

Figure 3: Numerical validation of the condition $B_{\ell}\left(y_{\mathrm{ac}}\right)>0$.

for these methods. We refer to [42] for a theoretical consideration for the efficiency of the residual based a posteriori estimator for a/c coupling methods.

4. We also validate numerically the assumption that $B_{\ell}\left(y_{\mathrm{ac}}\right)>0$ and plot the constants $\min B_{\ell}\left(y_{\mathrm{ac}}\right)$ in Fig. 3.

5. We list the minimums of the second order derivatives at all the a/c solutions we obtain in Table 1 . We find that, at least in the current experiment, the 'nearest neighbour' dominates so that our estimates of the stability constant remain positive which are not affected by the 'cross' nearest neighbour terms $\partial_{1-1} V(y)$.

We can conclude that our residual based a posteriori error estimators can be used to select quasi-optimal meshes for error of the deformation gradient of the force based methods, at least for this testing problem. 
Table 1: List of the values of the second-order derivatives.

\begin{tabular}{|c|c|c|}
\hline \multicolumn{2}{|c|}{ Type of derivatives } & $\min$ \\
\hline$\partial_{11} V\left(y_{\mathrm{ac}}\right)$ & $\partial_{-1-1} V\left(y_{\mathrm{ac}}\right)$ & 10.8833 \\
\hline$\partial_{1-1} V\left(y_{\mathrm{ac}}\right)$ & $\partial_{-11} V\left(y_{\mathrm{ac}}\right)$ & -0.2337 \\
\hline$\partial_{22} V\left(y_{\mathrm{ac}}\right)$ & $\partial_{-2-2} V\left(y_{\mathrm{ac}}\right)$ & -0.1653 \\
\hline$\partial_{12} V\left(y_{\mathrm{ac}}\right)$ & $\partial_{21} V\left(y_{\mathrm{ac}}\right)$ & 0.0092 \\
\hline$\partial_{1-2} V\left(y_{\mathrm{ac}}\right)$ & $\partial_{-21} V\left(y_{\mathrm{ac}}\right)$ & -0.0107 \\
\hline$\partial_{2-1} V\left(y_{\mathrm{ac}}\right)$ & $\partial_{-12} V\left(y_{\mathrm{ac}}\right)$ & -0.0107 \\
\hline$\partial_{-1-2} V\left(y_{\mathrm{ac}}\right)$ & $\partial_{-2-1} V\left(y_{\mathrm{ac}}\right)$ & 0.0092 \\
\hline$\partial_{2-2} V\left(y_{\mathrm{ac}}\right)$ & $\partial_{-22} V\left(y_{\mathrm{ac}}\right)$ & 0.0005 \\
\hline
\end{tabular}

\subsection{Model adaptivity}

Our second numerical experiment is motivated by the fact that in the first numerical experiment, the external residual dominates which is shown in Fig. 4. Therefore, we consider a problem with only internal error to further show the effectiveness of our analysis.

We set $N=1000$ for which we expect to 'better' show the effect of the blending. The external force $g_{\ell}$ to be

$$
g_{\ell}:=\left\{\begin{array}{cl}
-0.02\left(1+\frac{1}{2 \varepsilon \ell}\right), & -500 \leq \ell<0, \\
0.02\left(\frac{1}{2 \varepsilon \ell}-1\right), & 0<\ell \leq 500
\end{array}\right.
$$

whose magnitude fits this small system.

Again we use the cubic blending function as defined in (6.1) with different blending width, 5, 10 and 20, and the 'adaptive' blending width strategy . In addition to that, we also consider two different blending functions, which are linear and quintic splines defined

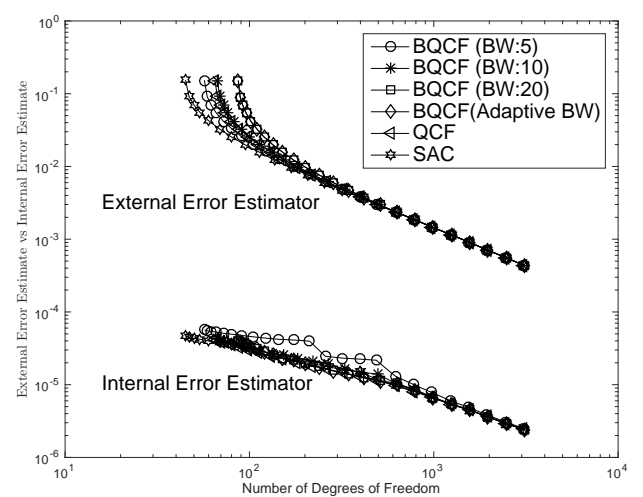

Figure 4: Comparison of the estimates of the external error and the internal error. 
by

$$
\begin{aligned}
& \hat{\beta}(x):= \begin{cases}0, & x<0, \\
x, & 0 \leq x \leq 1, \\
1, & x>1,\end{cases} \\
& \hat{\beta}(x):= \begin{cases}0, & x<0, \\
6 x^{5}-15 x^{4}+10 x^{3}, & 0 \leq x \leq 1, \\
1, & x>1,\end{cases}
\end{aligned}
$$

but fix the blending width to be 10 for these two cases. The relative errors of the strain gradient is again defined in (6.2).

\subsubsection{Adaptive algorithm}

In our computations, we begin with a mesh that is 'fully refined', which means each atom is a degree of freedom so that the external residual becomes 0 and only the internal residual plays the role. In this case, we consider the problem of adaptively choose the atomistic region, which is purely a model adaptivity problem. We note that similar problems was investigated in $[1,2]$ but for a different model and a different type of a posteriori error estimator. We use the nodewise error estimators defined in (3.4) and (3.14) to develop the following adaptive algorithm:

\section{Algorithm 6.2 A posteriori model adaptivity}

1. Create Mark the atoms $0, \pm \varepsilon, \pm 2 \varepsilon, \cdots,(\bar{K}-1) \varepsilon$ and $(\underline{K}+1) \varepsilon$ as atomistic. Create the blending region according to the blending width and mark the atoms outside the blending region to be continuous.

2. Solve: Compute the $\mathrm{QC}$ solution on the current decomposition of the lattice, compute the error indicators $\eta_{\ell}^{\text {ac }}$ for $\ell \in \mathscr{B} \cup \mathscr{C}$.

3. Mark: Choose a minimal subset $\mathscr{D} \subset\{1, \cdots, N\}$ of indices such that

$$
\sum_{\ell \in \mathscr{L}}\left(\eta_{\ell}^{\mathrm{ac}}\right)^{2} \geq \frac{1}{2} \sum_{\ell \in \mathscr{D}}\left(\eta_{\ell}^{\mathrm{ac}}\right)^{2}
$$

4. Refine: Mark all the atoms in $\mathscr{D}$ as atomistic and create the atomistic, blending and continuum regions accordingly.

5. Stop the algorithm if $\sum_{\ell \in \mathscr{L}}\left(\eta_{\ell}^{\mathrm{ac}}\right)<10^{-7}$; otherwise, go to Step (2).

\subsubsection{Numerical results}

We summarize the results of our numerical experiments. 


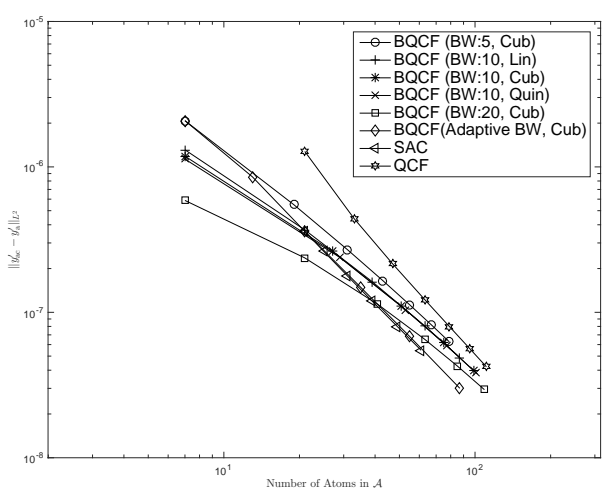

Figure 5: Relative errors in the deformation gradient plotted against the number of atoms in the atomistic region and the blending region.

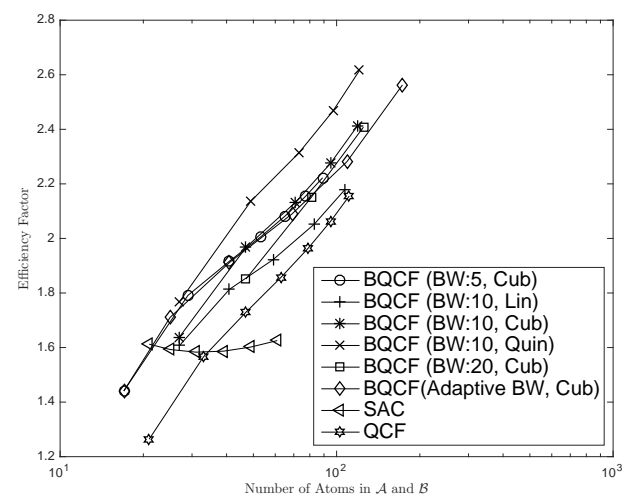

Figure 6: Efficiency factors plotted against the number of atoms in the atomistic and the blending region.

1. In Fig. 5, we display the gradient errors for the model adaptivity algorithms. We observe that the blending function has marginal effect on the accuracy of the solutions in this testing case whereas the blending width plays a more important role. This is somehow understandable since for a fixed $|\mathscr{A}|$, longer blending width means more atomistic information is taken into account. A notable fact is the superiority of the SAC method which achieves better accuracy with even less atomistic information which could be explained by the absence of any interface effect $[23,24]$. In that sense, the extension of the SAC method to higher dimension might be worth investigating.

2. We compute the efficiency factors which are shown in Fig. 6. The efficiency factors are again moderate. However, the efficiency factor is larger for the (B)QCF methods which might imply that our error estimator could be improved especially for treating the interface. 
We can conclude that our residual based a posteriori error estimators can also be used to select atomistic region for error of the deformation gradient. However, since we do not observe almost the same accuracy for all the methods we consider, we do not expect to develop model adaptivity methods for adaptively deciding the blending widths or selecting the blending functions using residual based error estimators. Further possible researches will be discussed in the next section.

\section{Conclusions}

We derive the a posteriori error estimators for the deformation gradient for three different force-based a/c methods for a periodic atomistic chain. Based on these estimators we propose mesh adaptive algorithms which adaptively choose location of the degrees of freedom and model adaptive algorithms which adaptively decide the locationof the atomistic region. Our numerical experiments show that our algorithms are quasi-optimal.

There are several possible future researches in this direction.

The first is the adaptive method for the BQCF methods which includes the adaptivity for the blending width and the blending function. Since prior analysis shows that the blending width and the blending function will substantially affect the stability of the method near instability $[14-16,32]$, we will pursue a further study in this direction together with a sharper a posteriori stability estimate.

The second is the adaptive method for the BQCE methods, including the adaptivity for the blending width and the blending function, using residual based estimators. We believe this is promising as the blending width and the blending function will affect the accuracy of the solution which should be detected by the residual based estimators. The difficulty there is to set up an appropriate optimization problem which takes the blending width and the blending function as input factors.

The third is the extension of adaptivity for a/c methods in higher dimensions. A notable progress has been made in [41] but we believe our analysis in 1D also provides an important stepping-stone in this direction.

\section{Appendix A Proof for lemma 4.1}

Proof. For any $y \in \mathscr{Y}^{\varepsilon}$, direct calculation gives us the following formula of second variation with arbitrary test function $v, w \in \mathscr{U}$ :

$$
\begin{gathered}
\left\langle\delta^{2} \mathscr{E}^{\mathrm{a}}(y) v, w\right\rangle=\varepsilon \sum_{\ell \in \mathscr{L}} \tilde{A}_{\ell}(y) w_{\ell-3}^{\prime} v_{\ell}^{\prime}+\tilde{B}_{\ell}(y) w_{\ell-2}^{\prime} v_{\ell}^{\prime}+\tilde{C}_{\ell}(y) w_{\ell-1}^{\prime} v_{\ell}^{\prime}+\tilde{D}_{\ell}(y) w_{\ell}^{\prime} v_{\ell}^{\prime} \\
+\tilde{E}_{\ell}(y) w_{\ell+1}^{\prime} v_{\ell}^{\prime}+\tilde{F}_{\ell}(y) w_{\ell+2}^{\prime} v_{\ell}^{\prime}+\tilde{G}_{\ell}(y) w_{\ell+3}^{\prime} v_{\ell}^{\prime}
\end{gathered}
$$


where

$$
\begin{aligned}
& \tilde{A}_{\ell}(y):= \partial_{2,-2}\left(D y_{\ell-2}\right) \\
& \tilde{B}_{\ell}(y):=-\left[\partial_{2,-1}\left(D y_{\ell-2}\right)+\partial_{2,-2}\left(D y_{\ell-2}\right)+\partial_{1,-2}\left(D y_{\ell-1}\right)+\partial_{2,-2}\left(D y_{\ell-1}\right)\right], \\
& \tilde{C}_{\ell}(y):=\partial_{21}\left(D y_{\ell-2}\right)+\partial_{22}\left(D y_{\ell-2}\right)-\partial_{1,-1}\left(D y_{\ell-1}\right)-\partial_{1,-2}\left(D y_{\ell-1}\right) \\
& \quad-\partial_{2,-1}\left(D y_{\ell-1}\right)-\partial_{2,-2}\left(D y_{\ell-1}\right)+\partial_{-1,-2}\left(D y_{\ell}\right)+\partial_{-2,-2}\left(D y_{\ell}\right), \\
& \tilde{D}_{\ell}(y):=\partial_{22}\left(D y_{\ell-2}\right)+\partial_{11}\left(D y_{\ell-1}\right)+2 \partial_{12}\left(D y_{\ell-1}\right)+\partial_{22}\left(D y_{\ell-1}\right)+\partial_{-1,-1}\left(D y_{\ell}\right) \\
& \quad+2 \partial_{-1,-2}\left(D y_{\ell}\right)+\partial_{-2,-2}\left(D y_{\ell}\right)+\partial_{-2,-2}\left(D y_{\ell+1}\right), \\
& \tilde{E}_{\ell}(y):=\partial_{12}\left(D y_{\ell-1}\right)+\partial_{22}\left(D y_{\ell-1}\right)-\partial_{-1,1}\left(D y_{\ell}\right)-\partial_{-1,2}\left(D y_{\ell}\right)-\partial_{-2,1}\left(D y_{\ell}\right) \\
& \quad-\partial_{-2,2}\left(D y_{\ell}\right)+\partial_{-2,-1}\left(D y_{\ell+1}\right)+\partial_{-2,-2}\left(D y_{\ell+1}\right), \\
& \tilde{F}_{\ell}(y):=-\left[\partial_{-1,2}\left(D y_{\ell}\right)+\partial_{-2,2}\left(D y_{\ell}\right)+\partial_{-2,1}\left(D y_{\ell+1}\right)+\partial_{-2,2}\left(D y_{\ell+1}\right)\right], \\
& \tilde{G}_{\ell}(y):=-\partial_{-2,2}\left(D y_{\ell+1}\right) .
\end{aligned}
$$

Before giving further analysis, we define some upper bounds for the second- and thirdorder derivatives of the potential function $V$ which will be used later. Let $g:=\left(g_{1}, g_{2}, g_{3}\right.$, $\left.g_{4}\right) \in \mathbb{R}^{1 \times 4}$ and $E^{\times 4}:=E \times 2 E \times-E \times-2 E$, we define

$$
\begin{array}{lll}
\underline{m}_{1}(E):=\max _{\boldsymbol{g} \in E^{\times 4}}\left\{\max _{(i, j) \in \mathscr{S}^{4}}\left|\partial_{i j} V(\boldsymbol{g})\right|\right\}, & \underline{m}_{2}(E):=\max _{\boldsymbol{g} \in E^{\times 4}}\left\{\max _{(i, j, k) \in \mathscr{S} \mathscr{S}^{4}}\left|\partial_{i j k} V(\boldsymbol{g})\right|\right\} ; \\
\bar{m}_{1}(E):=\max _{\boldsymbol{g} \in E^{\times 4}}\left\{\max _{(i, j) \in \mathscr{S}^{3}}\left|\partial_{i j} V(\boldsymbol{g})\right|\right\}, & \bar{m}_{2}(E):=\max _{\boldsymbol{g} \in E^{\times 4}}\left\{\max _{(i, j, k) \in \mathscr{S} \mathscr{S}^{3}}\left|\partial_{i j k} V(\boldsymbol{g})\right|\right\} ; \\
\underline{M}_{1}(E):=\max _{\boldsymbol{g} \in E^{\times 4}}\left\{\max _{(i, j) \in \mathscr{S}^{2}}\left|\partial_{i j} V(\boldsymbol{g})\right|\right\}, & \underline{M}_{2}(E):=\max _{\boldsymbol{g} \in E^{\times 4}}\left\{\max _{(i, j, k) \in \mathscr{S} \mathscr{S}^{2}}\left|\partial_{i j k} V(\boldsymbol{g})\right|\right\} ; \\
\bar{M}_{1}(E):=\max _{\boldsymbol{g} \in E^{\times 4}}\left\{\max _{(i, j) \in \mathscr{S}^{1}}\left|\partial_{i j} V(\boldsymbol{g})\right|\right\}, & \bar{M}_{2}(E):=\max _{\boldsymbol{g} \in E^{\times 4}}\left\{\max _{(i, j, k) \in \mathscr{S S S}^{1}}\left|\partial_{i j k} V(\boldsymbol{g})\right|\right\},
\end{array}
$$

where

$$
\begin{array}{rlrl}
\mathscr{S}^{1} & :=\{ \pm(1,1)\}, & & \mathscr{S} \mathscr{S}^{1}:=\{ \pm(1,1,1)\} ; \\
\mathscr{S}^{2}:=\{ \pm(1,-1), \pm(2,2)\}, & \mathscr{S} \mathscr{S}^{2}:=\{ \pm(1,1,-1), \pm(2,2,2)\} ; \\
\mathscr{S}^{3}:=\{ \pm(1,2), \pm(1,-2)\}, & \mathscr{S} \mathscr{S}^{3}:=\{ \pm(1,1,2), \pm(1,1,-2), \pm(1,2,2), \pm(1,-2,-2)\} ; \\
\mathscr{S}^{4}:=\{ \pm(2,-2)\}, & \mathscr{S} \mathscr{S}^{4}:=\{ \pm(1,2,-2), \pm(1,-1,-2), \pm(2,2,-2)\} .
\end{array}
$$

Remark A.1. We classify the partial derivatives as above in order for a sharper estimate. Numerical experiments show that: when $\mu$ and $v$ are close to $\nabla y_{\mathrm{a}}$, we have $\underline{m}_{j} \ll \bar{m}_{j} \ll$ $\underline{M}_{j} \ll \bar{M}_{j}$ for $j=1,2$. Therefore, we should avoid using $\bar{M}_{j}$, which is the uniform upper bounds for all derivatives, for every derivative to give an (quasi-) optimal estimate.

For any $\ell \in \mathscr{L}$, the following upper bounds hold that

$$
\begin{aligned}
& \left|\tilde{A}_{\ell}(y)\right|,\left|\tilde{G}_{\ell}(y)\right| \leq \underline{m}_{1}(E), \\
& \left|\tilde{B}_{\ell}(y)\right|,\left|\tilde{F}_{\ell}(y)\right| \leq 2\left[\underline{m}_{1}(E)+\bar{m}_{1}(E)\right], \\
& \left|\tilde{C}_{\ell}(y)\right|,\left|\tilde{E}_{\ell}(y)\right| \leq \underline{m}_{1}(E)+4 \bar{m}_{1}(E)+3 \underline{M}_{1}(E), \\
& \left|\tilde{D}_{\ell}(y)\right| \leq 4 \bar{m}_{1}(E)+4 \underline{M}_{1}(E)+2 \bar{M}_{1}(E) .
\end{aligned}
$$


and thus for any $y, z \in \mathscr{Y}^{\varepsilon}$, by (A.1) we have

$$
\begin{aligned}
& \left\langle\delta^{2} \mathscr{E}^{\mathrm{a}}(y) v, w\right\rangle-\left\langle\delta^{2} \mathscr{E}^{\mathrm{a}}(z) v, w\right\rangle \\
= & \varepsilon \sum_{\ell \in \mathscr{L}}\left(\tilde{A}_{\ell}(y)-\tilde{A}_{\ell}(z)\right) w_{\ell-3}^{\prime} v_{\ell}^{\prime}+\left(\tilde{B}_{\ell}(y)-\tilde{B}_{\ell}(z)\right) w_{\ell-2}^{\prime} v_{\ell}^{\prime} \\
& +\left(\tilde{C}_{\ell}(y)-\tilde{C}_{\ell}(z)\right) w_{\ell-1}^{\prime} v_{\ell}^{\prime}+\left(\tilde{D}_{\ell}(y)-\tilde{D}_{\ell}(z)\right) w_{\ell}^{\prime} v_{\ell}^{\prime} \\
& +\left(\tilde{E}_{\ell}(y)-\tilde{E}_{\ell}(z)\right) w_{\ell+1}^{\prime} v_{\ell}^{\prime}+\left(\tilde{F}_{\ell}(y)-\tilde{F}_{\ell}(z)\right) w_{\ell+2}^{\prime} v_{\ell}^{\prime}+\left(\tilde{G}_{\ell}(y)-\tilde{G}_{\ell}(z)\right) w_{\ell+3}^{\prime} v_{\ell}^{\prime} .
\end{aligned}
$$

We note that

$$
\begin{aligned}
& \left|\tilde{A}_{\ell}(y)-\tilde{A}_{\ell}(z)\right| \\
= & \left|\partial_{2,-2}\left(D y_{\ell-2}\right)-\partial_{2,-2}\left(D z_{\ell-2}\right)\right| \\
\leq & \left|\partial_{2,-2} V\left(y_{\ell-1}^{\prime}, y_{\ell-1}^{\prime}+y_{\ell}^{\prime},-y_{\ell-2}^{\prime},-y_{\ell-3}^{\prime}-y_{\ell-2}^{\prime}\right)-\partial_{2,-2} V\left(z_{\ell-1}^{\prime}, y_{\ell-1}^{\prime}+y_{\ell}^{\prime},-y_{\ell-2}^{\prime},-y_{\ell-3}^{\prime}-y_{\ell-2}^{\prime}\right)\right| \\
& +\left|\partial_{2,-2} V\left(z_{\ell-1}^{\prime}, y_{\ell-1}^{\prime}+y_{\ell}^{\prime},-y_{\ell-2}^{\prime},-y_{\ell-3}^{\prime}-y_{\ell-2}^{\prime}\right)-\partial_{2,-2} V\left(z_{\ell-1}^{\prime}, z_{\ell-1}^{\prime}+z_{\ell}^{\prime},-y_{\ell-2}^{\prime},-y_{\ell-3}^{\prime}-y_{\ell-2}^{\prime}\right)\right| \\
& +\left|\partial_{2,-2} V\left(z_{\ell-1}^{\prime}, z_{\ell-1}^{\prime}+z_{\ell}^{\prime},-y_{\ell-2}^{\prime},-y_{\ell-3}^{\prime}-y_{\ell-2}^{\prime}\right)-\partial_{2,-2} V\left(z_{\ell-1}^{\prime}, z_{\ell-1}^{\prime}+z_{\ell}^{\prime},-z_{\ell-2}^{\prime},-y_{\ell-3}^{\prime}-y_{\ell-2}^{\prime}\right)\right| \\
& +\left|\partial_{2,-2} V\left(z_{\ell-1}^{\prime}, z_{\ell-1}^{\prime}+z_{\ell}^{\prime},-z_{\ell-2}^{\prime},-y_{\ell-3}^{\prime}-y_{\ell-2}^{\prime}\right)-\partial_{2,-2} V\left(z_{\ell-1}^{\prime}, z_{\ell-1}^{\prime}+z_{\ell}^{\prime},-z_{\ell-2}^{\prime},-z_{\ell-3}^{\prime}-z_{\ell-2}^{\prime}\right)\right| .
\end{aligned}
$$

Applying the mean value theorem we have

$$
\begin{aligned}
& \quad\left|\tilde{A}_{\ell}(y)-\tilde{A}_{\ell}(z)\right| \\
& \leq \max _{\boldsymbol{g} \in E^{\times 4}}\left|\partial_{2,-2,1} V(\boldsymbol{g})\right|\left|y_{\ell-1}^{\prime}-z_{\ell-1}^{\prime}\right|+\max _{\boldsymbol{g} \in E^{\times 4}}\left|\partial_{2,-2,2} V(\boldsymbol{g})\right|\left[\left|y_{\ell-1}^{\prime}-z_{\ell-1}^{\prime}\right|+\left|y_{\ell}^{\prime}-z_{\ell}^{\prime}\right|\right] \\
& \quad+\max _{\boldsymbol{g} \in E^{\times 4}}\left|\partial_{2,-2,-1} V(\boldsymbol{g})\right|\left|y_{\ell-2}^{\prime}-z_{\ell-2}^{\prime}\right| \\
& \quad+\max _{\boldsymbol{g} \in E^{\times 4}}\left|\partial_{2,-2,-2} V(\boldsymbol{g})\right|\left[\left|y_{\ell-3}^{\prime}-z_{\ell-3}^{\prime}\right|+\left|y_{\ell-2}^{\prime}-z_{\ell-2}^{\prime}\right|\right] \\
& \leq 6 \underline{m}_{2}(E)|| y^{\prime}-z^{\prime} \|_{\ell_{\varepsilon}^{\infty}}
\end{aligned}
$$

Similarly, we have

$$
\begin{aligned}
& \left|\tilde{G}_{\ell}(y)-\tilde{G}_{\ell}(z)\right| \leq 6 \underline{m}_{2}(E)\left\|y^{\prime}-z^{\prime}\right\|_{\ell_{\varepsilon}^{\infty}}, \\
& \left|\tilde{B}_{\ell}(y)-\tilde{B}_{\ell}(z)\right|,\left|\tilde{F}_{\ell}(y)-\tilde{F}_{\ell}(z)\right| \leq\left[18 \underline{m}_{2}(E)+6 \bar{m}_{2}(E)\right]\left\|y^{\prime}-z^{\prime}\right\|_{\ell_{\varepsilon}^{\infty}}, \\
& \left|\tilde{C}_{\ell}(y)-\tilde{C}_{\ell}(z)\right|,\left|\tilde{E}_{\ell}(y)-\tilde{E}_{\ell}(z)\right| \leq\left[26 \underline{m}_{2}(E)+16 \bar{m}_{2}(E)+6 \underline{M}_{2}(E)\right]\left\|y^{\prime}-z^{\prime}\right\|_{\ell_{\varepsilon}^{\infty}}, \\
& \left|\tilde{D}_{\ell}(y)-\tilde{D}_{\ell}(z)\right| \leq\left[20 \underline{m}_{2}(E)+28 \bar{m}_{2}(E)+10 \underline{M}_{2}(E)+2 \bar{M}_{2}(E)\right]\left\|y^{\prime}-z^{\prime}\right\|_{\ell_{\varepsilon}^{\infty}} .
\end{aligned}
$$

We may then find an upper bound for (A.4) as

$$
\begin{aligned}
& \left|\left\langle\delta^{2} \mathscr{E}^{\mathrm{a}}(y) v, w\right\rangle-\left\langle\delta^{2} \mathscr{E}^{\mathrm{a}}(z) v, w\right\rangle\right| \\
\leq & {\left[2 \bar{M}_{2}(E)+22 \underline{M}_{2}(E)+72 \bar{m}_{2}(E)+120 \underline{m}_{2}(E)\right]\left\|y^{\prime}-z^{\prime}\right\|_{\ell_{\varepsilon}^{\infty}}\left\|w^{\prime}\right\|_{\ell_{\varepsilon}^{2}}\left\|v^{\prime}\right\|_{\ell_{\varepsilon}^{2}} } \\
= & : C_{\mathrm{Lip}}\left\|y^{\prime}-z^{\prime}\right\|_{\ell_{\varepsilon}^{\infty}}\left\|w^{\prime}\right\|_{\ell_{\varepsilon}^{2}}\left\|v^{\prime}\right\|_{\ell_{\varepsilon}^{2}} .
\end{aligned}
$$

Therefore, $C_{\text {Lip }}$ can be chosen as $C_{\text {Lip }}=2 \bar{M}_{2}(E)+22 \underline{M}_{2}(E)+72 \bar{m}_{2}(E)+120 \underline{m}_{2}(E)$, which concludes the proof of Lemma 4.1. 
Acknowledgments Hao Wang was partially supported by NSFC grant 11501389, 114712 14 and Sichuan University Starting Up Research Funding No. 2082204194117. Shaohui Liu was partially supported by Sichuan University National Students' Platform for Innovation and Entrepreneurship Training Program No. 201610610172 and Stony Brook University PhD Scholarship.

\section{References}

[1] M. ARndt AND M. Luskin, Goal-oriented atomistic-continuum adaptivity for the quasicontinuum approximation, Int. J. Multiscale Comput. Engrg., 5(49-50) (2007), pp. 407-415.

[2] M. ARNDT AND M. Luskin, Error estimation and atomistic-continuum adaptivity for the quasicontinuum approximation of a Frenkel-Kontorova model, Multiscale Model. Simul., 7(1) (2008), pp. 147-170.

[3] M. ARNDT AND M. Luskin, Goal-oriented adaptive mesh refinement for the quasicontinuum approximation of a Frenkel-Kontorova model, Comput. Methods Appl. Mech. Engrg., 197(49-50) (2008), pp. 4298-4306.

[4] M. Luskin, M. Dobson And C. ORTner, Sharp stability estimates for the force-based quasicontinuum approximation of homogeneous tensile deformation, Multiscale Model. Simul., 8(3) (2010), pp. 782-802.

[5] M. Luskin, M. Dobson And C. Ortner, Iterative methods for the force-based quasicontinuum approximation: Analysis of a 1d model problem, Comput. Methods Appl. Mech. Engrg., 200 (2011), pp. 2697-2709.

[6] M. Dobson AND M. Luskin, Analysis of a force-based quasicontinuum approximation, M2AN Math. Model. Numer. Anal., 42(1) (2008), pp. 113-139.

[7] M. Dobson And M. Luskin, An analysis of the effect of ghost force oscillation on the quasicontinuum error, M2AN Math. Model. Numer. Anal., 43(3) (2009), pp. 591-604.

[8] M. Dobson, M. Luskin And C. Ortner, Accuracy of quasicontinuum approximations near instabilities, J. Mech. Phys. Solids, 58 (2010), pp. 1741-1757.

[9] M. Dobson, M. Luskin AND C. ORTnER, Stability, instability, and error of the force-based quasicontinuum approximation, Arch. Ration. Mech. Anal., 197(1) (2010), pp. 179-202.

[10] C. Ortner, M. Dobson And A. V. Shapeev, The spectrum of the force-based quasicontinuum operator for a homogeneous periodic chain, Multiscale Model. Simul., 10(3) (2012), pp. 744 765.

[11] W. DöRfler, A convergent adaptive algorithm for poisson's equationčň SIAM J. Numer. Anal., 33 (1996), pp. 1106-1124.

[12] E. Weinan, J. Lu AND J. Z. YAng, Uniform accuracy of the quasicontinuum method, Mrs Proceedings, 978(21) (2006), pp. 4070-4079.

[13] V. Ehrlacher, C. Ortner and A. V. Shapeev, Analysis of boundary conditions for crystal defect atomistic simulations, Arch. Ration. Mech. Anal., 222 (2016), pp. 1217-1268.

[14] M. Luskin, C. ORTNER, X. Li AND A. V. SHAPEEv, Theory-based benchmarking of the blended forcebased quasicontinuum method, Comput. Methods Appl. Mech. Engrg., 268 (2014), pp. $763-$ 781.

[15] C. Ortner, A. V. Shapeev, X. Li And B. V. Koten, Analysis of blended atomistic/continuum hybrid methods, Numer. Math., 134 (2016), pp. 275-326.

[16] X. Li, M. Luskin AND C. ORTNER, Positive-definiteness of the blended force-based quasicontinuum method, Multiscale Model. Simul., 10(3) (2012), pp. 1023-1045. 
[17] X. H. Li AND M. Luskin, A generalized quasinonlocal atomistic-to-continuum coupling method with finite-range interaction, IMA J. Num. Anal., 32(2) (2012), pp. 373-393.

[18] P. Lin, Theoretical and numerical analysis for the quasi-continuum approximation of a material particle model, Math. Comp., 72(242) (2003), pp. 657-675.

[19] P. LIN, Convergence analysis of a quasi-continuum approximation for a two-dimensional material without defects, SIAM J. Numer. Anal., 45(1) (2007), pp. 313-332.

[20] P. Lin AND A. Shapeev, Energy-based ghost force removing techniques for the quasicontinuum method, Mathematics, 2009.

[21] J. Lu AND P. Ming, Convergence of a force-based hybrid method with planar sharp interface, Siam J. Numer. Anal, 52 (2014), pp. 2005-2026.

[22] J. Lu AND P. Ming, Stability of a force-based hybrid method with planar sharp interface, SIAM J. Numer. Anal., 52(4) (2014), pp. 2005-2026.

[23] C. Makridakis, C. Ortner and E. Süli, Stress-based atomistic/continuum coupling: a new variant of the quasicontinuum approximation, Int. J. Multiscale Com., 10(1) (2012), pp. 5164.

[24] C. Makridakis, C. Ortner and E. Süli, A priori error analysis of two force-based atomistic/continuum models of a periodic chain, Numerische Mathematik, 2011, pp. 1-39.

[25] R. E. Miller And E. B. TADMOR, The quasicontinuum method: Overview, applications and current directions, J. Computer-Aided Mater., 9 (2003), pp. 203-239.

[26] R. E. Miller And E. B. TADMOR, A unified framework and performance benchmark of fourteen multiscale atomistic/continuum coupling methods, Modelling Simul. Mater. Sci. Eng., 17(5) (2009), 053001.

[27] P. Ming And J. Lu, Convergence of a force-based hybrid method in three dimensions, Comm. Pure Appl. Math, 66 (2013), pp. 83-108.

[28] P. Ming AND J. YANG, Analysis of a one-dimensional nonlocal quasi-continuum method, Multiscale Model. Simul., 7(4) (2009), pp. 1838-1875.

[29] P. Morin, R. H. NochetTo AND K. G. Siebert, Data oscillation and convergence of adaptive fem, SIAM J. Numer. Anal., 38 (2000), pp. 466-488.

[30] M. Ortiz, R. Phillips and E. B. TAdmor, Quasicontinuum analysis of defects in solids, Philosophical Magazine A, 73(6) (1996), pp. 1529-1563.

[31] C. ORtner, A priori and a posteriori analysis of the quasinonlocal quasicontinuum method in 1D, Math. Comp., 80(275) (2011), pp. 1265-1285.

[32] C. Ortner And M. Luskin, Atomistic-to-continuum coupling, Acta Numerica, 22 (2013), pp. 397-508.

[33] C. ORTNER AND H. WANG, A priori error estimates for energy-based quasicontinuum approximations of a periodic chain, Math. Models Methods Appl. Sc., 21 (2011), pp. 2491-2521.

[34] C. ORTNER AND H. WANG, A posteriori error control for a quasi-continuum approximation of a periodic chain, IMA J. Num. Anal., 34(3) (2014), pp. 977-1001.

[35] C. Ortner AND L. Zhang, Construction and sharp consistency estimates for atomistic/continuum coupling methods with general interfaces: A $2 d$ model problem, SIAM J. Numer. Anal., 50(6) (2012), pp. 2940-2965.

[36] C. ORTNER AND L. ZhANG, Energy-based atomistic-to-continuum coupling without ghost forces, Comput. Methods Appl. Mech. Engrg., 279 (2014), pp. 29-45.

[37] S. Prudhomme, L Chamoin, H. B. Dhita and P. T. Bauman, An adaptive strategy for the control of modeling error in two-dimensional atomic-to-continuum coupling simulations, Comput. Method. Appl. M., 198(21-26) (2009), pp. 1887-1901.

[38] A. V. SHAPEEv, Consistent energy-based atomistic/continuum coupling for two-body potentials in one and two dimensions, Multiscale Model. Simul., 9(3) (2011), pp. 905-932. 
[39] A. V. Shapeev, Consistent energy-based atomistic/continuum coupling for two-body potentials in three dimensions, SIAM J. Sci. Comput., 34(3) (2012), pp. 335-360.

[40] T. Shimokawa, J. J. Mortensen, J. Schiøtz and K. W. Jacobsen, Matching conditions in the quasicontinuum method: Removal of the error introduced at the interface between the coarsegrained and fully atomistic region, Phys. Rev. B, 69(21) (2004), pp. 148-152.

[41] H. WAng, M. Liao, P. Lin And L. Zhang, A Posteriori Error Estimation and Adaptive Algorithm for the Atomistic/Continuum Coupling, 2nd Ed., ArXiv e-prints, 1702.02701v1, 2017.

[42] H. Wang and S. Zhang, Analysis of the Residual Type and the Recovery Type a Psoteriori Error Estimators for a Consistent Atomistic-to-Continuum Coupling Method, 1st Ed., ArXiv e-prints, 1702.04048v3, 2017. 